\title{
Multiplexed Profiling of Extracellular Vesicles for Biomarker Development
}

Cite as

Nano-Micro Lett.

(2022) 14:3

Received: 8 August 2021

Accepted: 22 October 2021

Published online: 2 December 2021

(C) The Author(s) 2021

\author{
Cheng Jiang ${ }^{1,2,5}$, Ying $\mathrm{Fu}^{2}$, Guozhen $\mathrm{Liu}^{3}$, Bowen Shu ${ }^{4}$, Jason Davis ${ }^{2}$, \\ George K. Tofaris 1,5 凶
}

\section{HIGHLIGHTS}

- Extracellular vesicle (EV) multiplexing involves chemical, physical spatial, biological, or nanoparticle-based strategies.

- Multiplexing in EV biomarker development includes high-throughput screening as well as point-of-care testing platforms which to date have been applied mainly to EV surface proteins or internal cargo miRNAs.

- Multiplexed measurements at single-EV resolution are likely to revolutionize the applicability of EV analytes as biomarkers in complex and heterogeneous diseases.

\begin{abstract}
Extracellular vesicles (EVs) are cell-derived membranous particles that play a crucial role in molecular trafficking, intercellular transport and the egress of unwanted proteins. They have been implicated in many diseases including cancer and neurodegeneration. EVs are detected in all bodily fluids, and their protein and nucleic acid content offers a means of assessing the status of the cells from which they originated. As such, they provide opportunities in biomarker discovery for diagnosis, prognosis or the stratification of diseases as well as an objective monitoring of therapies. The simultaneous assaying of multiple EV-derived markers will be required for an impactful practical application, and multiplexing platforms have evolved with the potential to achieve this. Herein, we provide a comprehensive overview of the currently available multiplexing platforms for EV analysis, with a primary focus on miniaturized and integrated devices that offer potential step changes in analytical power, throughput and consistency.
\end{abstract}

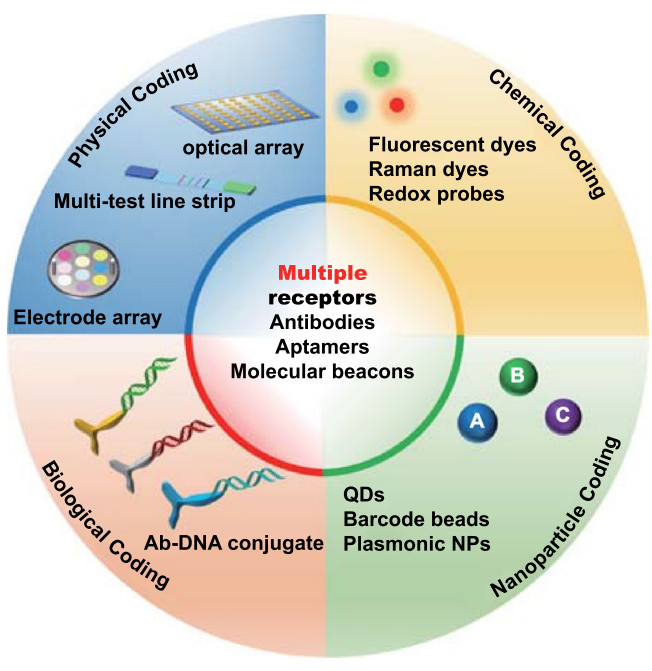

KEYWORDS Multiplexed profiling; Extracellular vesicles; Exosomes; Liquid biopsy; Point-of-care; Biomarker

\footnotetext{
Cheng Jiang, cheng.jiang@ndcn.ox.ac.uk; Jason Davis, jason.davis@chem.ox.ac.uk; George K. Tofaris, george.tofaris@ndcn.ox.ac.uk

Nuffield Department of Clinical Neurosciences, New Biochemistry Building, University of Oxford, Oxford OX1 3QU, UK

2 Department of Chemistry, University of Oxford, Oxford OX1 3QZ, UK

3 School of Life and Health Sciences, The Chinese University of Hong Kong, Shenzhen 518172, People's Republic of China

4 Dermatology Hospital, Southern Medical University, Guangzhou 510091, People's Republic of China

5 Kavli Institute for Nanoscience Discovery, New Biochemistry Building, University of Oxford, Oxford, UK
} 


\section{Introduction}

Circulating extracellular vesicles (EVs) are a heterogeneous group of vesicles secreted by a variety of cell types, present in most bodily fluids (e.g. blood, saliva, urine, ascites, cerebrospinal fluid) and thought to function in intercellular communication by transferring cargo molecules from donor to recipient cells or facilitating the jettison of unwanted molecules to the cell exterior [1,2]. A large fraction of EVs consists of exosomes (30-150 nm in diameter), which are endosome-derived $\mathrm{EV}$ or ectosomes $(0.1-1.0 \mu \mathrm{m})$ derived from the plasma membrane. EVs carry parental cell-derived fingerprint proteins and RNAs but can also envelop diseaseassociated proteins and microRNAs [3, 4]. As such they are diagnostically relevant to cancers [5-10], neurodegenerative diseases [11-15], and cardiovascular disease [16, 17]. The potential applications of EV analysis in diagnosis and prognosis, are, then, substantial (Fig. 1a), especially if multiple targets can be screened simultaneously [18-20]. In this regard, multiplexed profiling has the advantage of increasing diagnostic accuracy and reducing sample volume and assay time as well as variability arising from the repeated processing of multiple sample aliquots for serial singleplexing. Due to the heterogeneity and complexity of human diseases, it is becoming clear that a single biomarker is unlikely to offer an accurate estimate of either a pathological state or phase. An efficient assessment of combinatorial markers has emerged as a promising way of measuring diseases with complex multifactorial aetiologies, and ultimately developing objective readouts for prognosis, stratification or therapy monitoring (Fig. 1b, c) [21-23].

Prior to any downstream analysis, EVs need to be isolated from complex biofluids. This can be achieved by utilizing their physical properties (e.g. size, density, surface charge) or surface markers as reviewed elsewhere [24-26]. Here we aim to provide a comprehensive overview of the fundamental strategies used to build multiplexing platforms for intact EVs and EV-derived proteins, RNAs, metabolites, and their application to clinical samples. We have also included platforms that have not yet been applied to EVs but could be

(a)

\section{Multiple EV analytes}

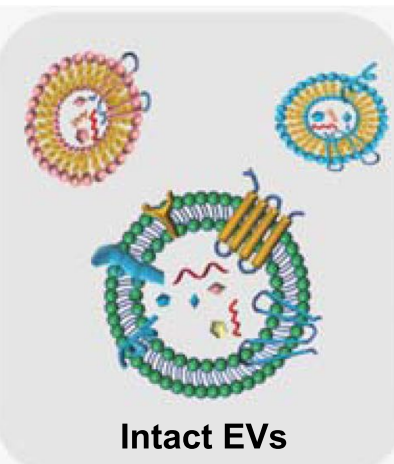

(b)

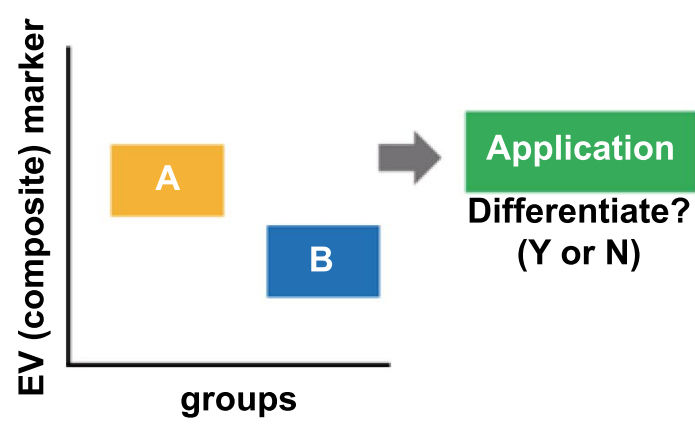

(c)

\begin{tabular}{|c|c|c|}
\hline A & B & Application \\
\hline $\begin{array}{c}\text { Patients } \\
\text { Before }\end{array}$ & Healthy control & Early diagnosis \\
\hline Disease A & Disease B & Therapy monitoring \\
\hline $\begin{array}{c}\text { Disease stage } \\
\text { T1 }\end{array}$ & $\begin{array}{c}\text { Disease stage } \\
\text { T2 }\end{array}$ & $\begin{array}{c}\text { Disease stratification } \\
\text { disease with similar } \\
\text { symptoms) }\end{array}$ \\
\hline
\end{tabular}

Fig. 1 Potential clinical applications of composite EV biomarkers. a The molecular profiling of EVs may be based on proteomics (e.g. membrane proteins and internal proteins), RNAs or metabolites (e.g. lipids and glycans). b The multiplexed analysis of EV components can generate a box plot representation of expression levels in A and B groups as defined in c. c Potential clinical applications of EV markers 
adapted for such purpose and discuss how this technology may evolve in the future.

\section{Overview of EV Multiplexed Profiling Strategies}

The multiplexed profiling of EVs should be considered as the capability of one detection platform to assay multiple EV-derived analytes, typically proteins, RNAs and metabolites in a short time scale. The innate requirement for each analyte to generate a distinguishable signal can be achieved by two generic strategies, i.e. an intrinsic fingerprint-based strategy (internal coding) or an external coding-based strategy (external coding). The former strategy employs native properties such as charge/mass $(\mathrm{m} / \mathrm{z})$ based mass spectroscopy, as reviewed elsewhere [27, 28]. The latter, as shown in Fig. 2, typically involves the use of multiple receptors (e.g. antibodies, aptamers, molecular beacons, or locked nucleic acids, LNA) as recognition components, in association with multiple reporters, e.g. chemical labels (optical dyes, redox probes), DNA oligonucleotides, or nanoparticle tags [29], for distinct signal generation derived from the binding events

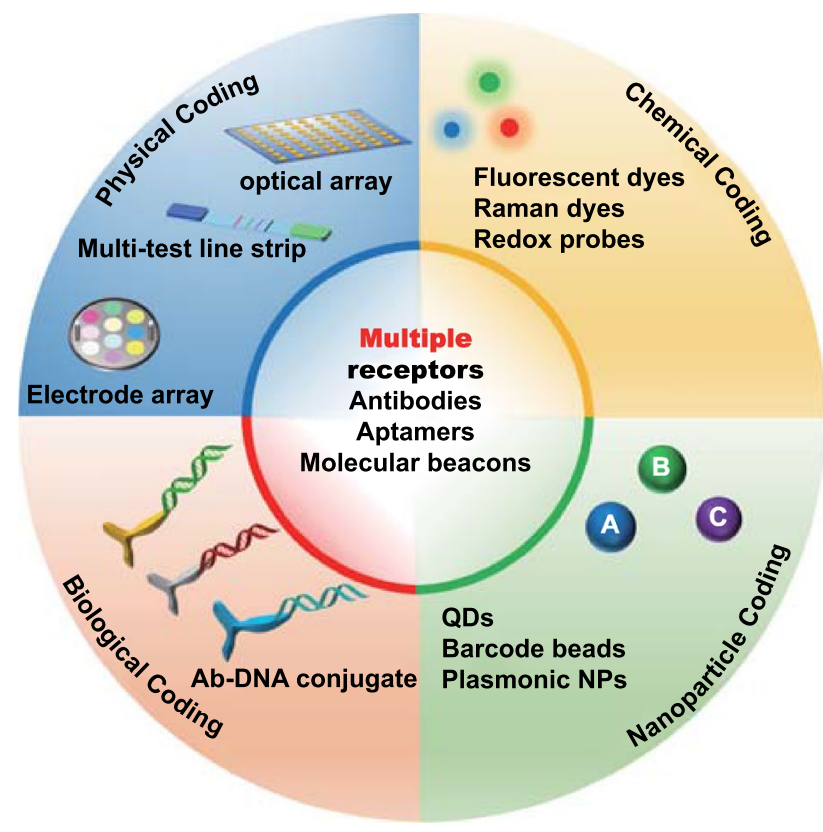

Fig. 2 Main external-coding strategies for EV profiling. Multiplexing is typically based on the combination of multiple receptors with one of the four coding strategies. Depending on how the analyte signal is generated and transduced, multiple external codes generated by chemical reporter labelling, physical spatial coding, biological coding, or nanoparticle coding, can be used in association with multiple receptors $(\mathrm{QDs}=$ quantum dots, $\mathrm{NP}=$ nanoparticles $)$ of multiple EV analytes and/or physically isolated regions of a solid interface such as those within multi-spot optical arrays or electrochemical arrays (i.e., position coding). Accordingly, the EV multiplexed profiling strategies can be divided into four main categories, chemical-, physical-, biological- or nanoparticle-based coding. In practice, this is achieved by bio-affinity induced binding events between receptor and EV analyte, potentially through the cooperation of multiple receptors, and using one of these four strategies or their combination. These will each be systematically discussed in the following sections. Generally, one receptor triggers one 'code' with the generation of multiple distinct codes enabling multiplexing.

\section{Chemical Coding}

For chemical coding, a range of small chemical reporters such as redox probes, fluorescent dyes and Raman tags can be employed in cooperation with capture ligands (i.e. receptors) to generate distinguishable and specific readouts for EV components. A broad range of methods are available to facilitate this, as detailed below. In this mode, different receptors, each matched with a chemical tag, bind to specific EV analytes, with each binding event generating a distinct readout. A detection of each $\mathrm{EV}$ analyte, i.e. the binding event of EV analyte with the corresponding code, is thus transduced into a signal for that code.

\subsection{Raman Tags}

Raman spectroscopy permits the resolution of unique molecular fingerprints based on vibrational and rotational modes. It reflects the overall chemical bond characteristics of EV molecular fingerprints including proteins, lipids, and metabolites. The resulting spectroscopic patterns are potentially able to differentiate between the origins of EVs or disease subgroups. To bypass the natively low signal/noise ratio of Raman spectroscopy, surface-enhanced Raman spectroscopy (SERS) makes use of the strong electromagnetic fields generated at appropriately designed plasmonic substrates [30-34]. For example, a correlation between nonsmall cell lung cancer (NSCLC) cell-derived exosomes and protein markers was shown through their unique Raman scattering 
profiles and subsequent principal component analysis (PCA) (Fig. 3a) [35].

Such native Raman spectra reflect overall intrinsic EV chemical components, whereas specific probing of EV components using multiple Raman tag labels can be applied using a SERS system. In this way, signals from different EV analytes can be transduced using the characteristic peaks of the corresponding Raman tag. For example, Zhang et al. have very recently studied the molecular profile of CD63-positive EVs using multiple SERS tags. In this work, EVs from three

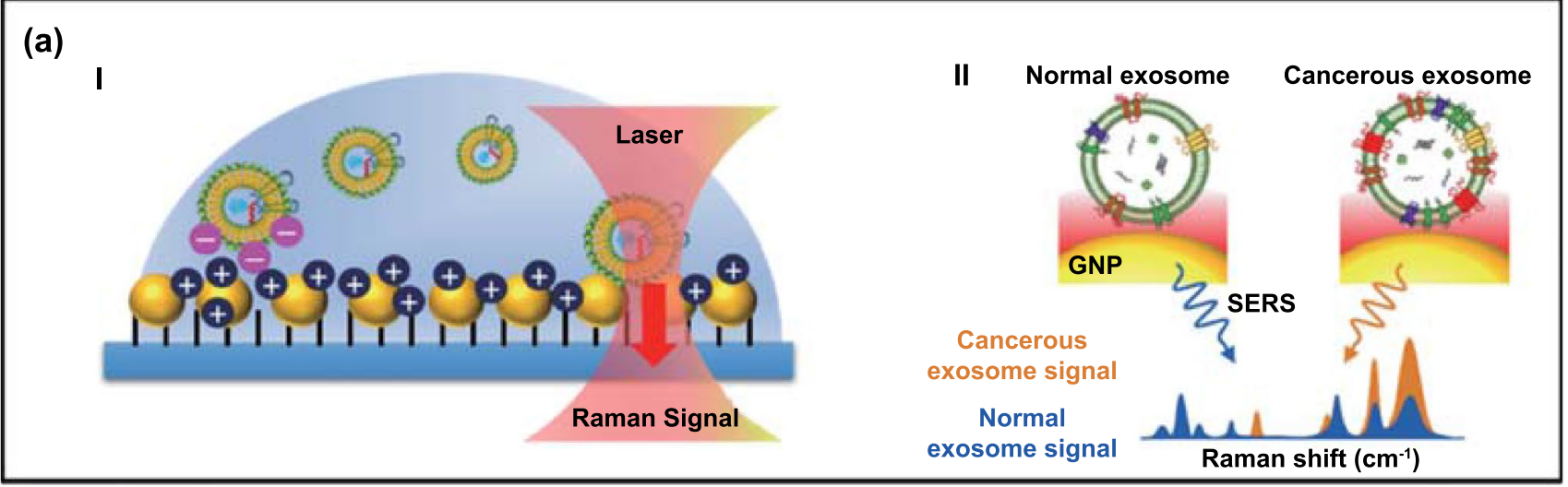

(b) CD44V6-TFMBA@AuNP
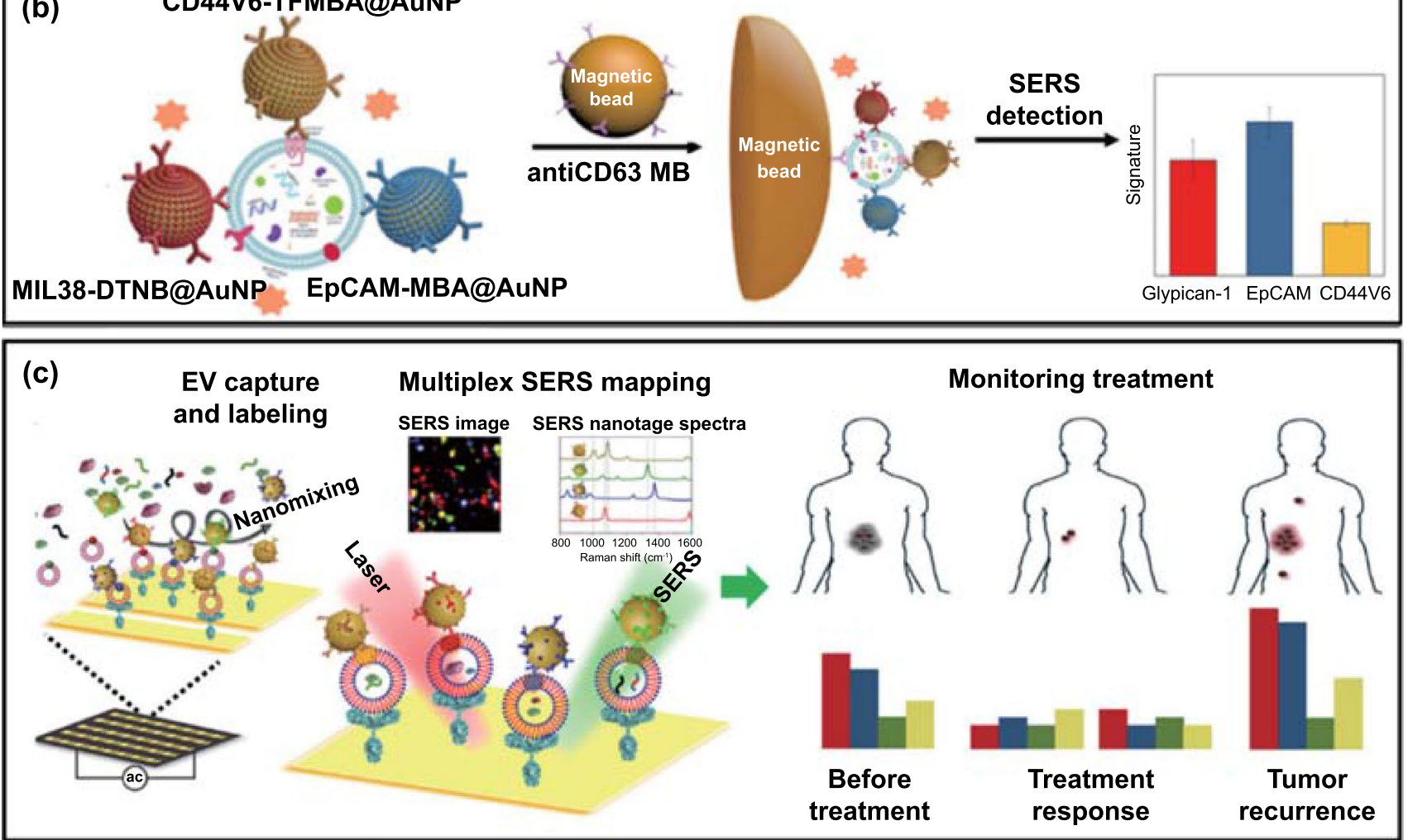

Fig. 3 Typical SERS-based approaches for EV multiplexing. a An examination of multiple EV components using the bulk chemical fingerprints of immobilized EVs. Adapted with permission from Ref. [35]. Copyright 2018 American Chemical Society. b Schematic illustration of molecular phenotype profiling of CD63-positive EVs using SERS nanotags (antibody-Raman dye conjugate: anti-MIL38-DTNB, anti-EpCAM-MBA, and anti-CD44V6-TFMBA). Adapted with permission from Ref. [36]. Copyright 2020 American Chemical Society. TFMBA: 2,3,5,6-Tetrafluoro-4-mercaptobenzonic acid, DTNB: 5,5'-dithiobis(2-nitrobenzoic acid), MBA: 4-mercaptobenzoic acid. c A multiplex EV phenotype assay chip using four SERS nanotags. The phenotypic evolution can be tracked by analysing EV samples before, during, and after immunotherapy treatment, thus providing information on treatment responses and the early signs of drug resistance. Adapted with permission from Ref. [39]. Copyright 2020 American Association for the Advancement of Science (AAAS) 
different cancer types were captured with anti-CD63 magnetic beads, followed by the addition of three types of SERS nanotags (i.e. Raman reporter-detection antibody-decorated AuNPs targeting the EV membrane proteins glypican-1, EpCAM, and CD44). In this way, a composite of magnetic beads-EVstriplex SERS nanotags was used for multiplexed detection (Fig. 3b) [36] and applied to EV proteins derived from cancer cell lines and plasma from patients with colorectal, bladder or pancreatic cancer. In similar work, aptamer-decorated magnetic beads as capture agents coupled with Raman dye conjugated aptamers@AuNPs [37] or gold nanorods (AuNRs) [38] were used as detection probes to profile specific membrane proteins from cancer-derived EVs. Wang et al. recently elaborated upon the multiplexed coding strategy by introducing four distinct SERS nanotags, i.e. AuNPs decorated with different antibodies (targeting surface proteins) and Raman reporters. Instead of using magnetic particles for EV capture, they generated anti-CD63-modified gold chips for the capture of generic EVs from plasma of melanoma patients and healthy controls (Fig. 3c) [39], followed by the addition of Raman dyeantibody@AuNPs based SERS probes against four membrane proteins. This study, in a small group of patients, demonstrated the potential of the technology in differentiating between healthy and disease states or monitoring the response to targeted therapies. It is noteworthy that, for such SERS analyses, a comprehensive consideration of $\mathrm{EV}$ dimensions and the size of the magnetic and gold components is necessary to avoid steric hindrance, which could affect the binding efficiency and thus the detection limit [40].

The combination of metallic substrates (planar or colloidal), receptors (e.g. antibodies, aptamers) and Raman dye-based SERS probes offers, then, one avenue for EV multiplexing, especially in the analysis of EV membrane proteins. A tuning of metallic structure can improve sensitivity such that multiplexing at single-EV level $[41,42]$ is possible. However, the poor reproducibility of fabrication of SERS substrate limits the clinical application of this method. A high-throughput fabrication using lithography, and the use of intrinsic EV Raman signatures for normalization may offer a solution to overcome such limitations.

\subsection{Fluorescent Dyes}

Besides Raman dyes, fluorescent dye-based chemical coding strategies have also been widely used for EV multiplexed profiling. This is generally achieved through the capturing of EVs on a specific receptor-modified flat or spherical interface, followed by the addition of multiple fluorescence dye-tagged ligands (e.g. antibodies, aptamers). For example, an integrated magnetic microfluidic chip with immunofluorescence has been developed by Mei and co-workers (Fig. 4a; termed the ExoSearch biochip) [43]. The magnetic bead-based microfluidic mixing-capture format offers several advantages, including rapid magnetic solid-phase isolation, flexible capture capacity, and good scalability [44-47]. In this work, plasma samples and anti-CD9 conjugated magnetic beads (MB) were injected into separate channels and, after on-chip mixing, MB-bound EVs were retained by a magnetic field followed by a multiplexed fluorescence assay using a panel of three fluorescently labelled antibodies targeting CA-125, EpCAM and CD24. The authors demonstrated high performance of the receiver operating characteristic (ROC) curve (ovarian cancer $v s$ healthy control) with an area under curve (AUC) $=1$ using either CA-125 or EpCAM as signature, and $\mathrm{AUC}=0.91$ for $\mathrm{CD} 24$. In a similar approach, Fang et al. recently developed a continuous-flow microfluidic system for the immunomagnetic capture of CD63(+) exosomes and detection of two exosomal tumour markers (EpCAM and HER2) in plasma samples using an immunofluorescence approach [48]. They demonstrated that EpCAM levels were significantly higher in exosomes from breast cancer patients compared to healthy controls. The scalability, multiplex capability, and potential for automation of the proposed magnetic microfluidics, together with larger validation cohort size and a composite marker format, may enhance its clinical translation. The integration of a chemical lysis step in another microfluidic chip enabled the quantification of a range of membrane-associated (EpCAM, $\alpha$-IGF-1R, CA125, CD9, CD81, and CD63) and internal (e.g. phosphorylated type 1 insulin growth factor receptor, IGF-1R) EV proteins from two lung cancer patients, two ovarian cancer patients, and healthy controls [49].

The examples mentioned above, focused on protein quantification with bulk EV preparations that are often heterogeneous. Single-EV analyses could, in principal, offer a more granular resolution of EV subpopulations [50]. In this regard, Lee et al. have recently explored a multiplexed fluorescent imaging system for assaying markers at single-EV resolution (Fig. 4b) [51]. In their study, the EVs were biotinylated and subsequently captured on a neutravidin-coated glass within a microfluidic chamber. The immobilized EVs 

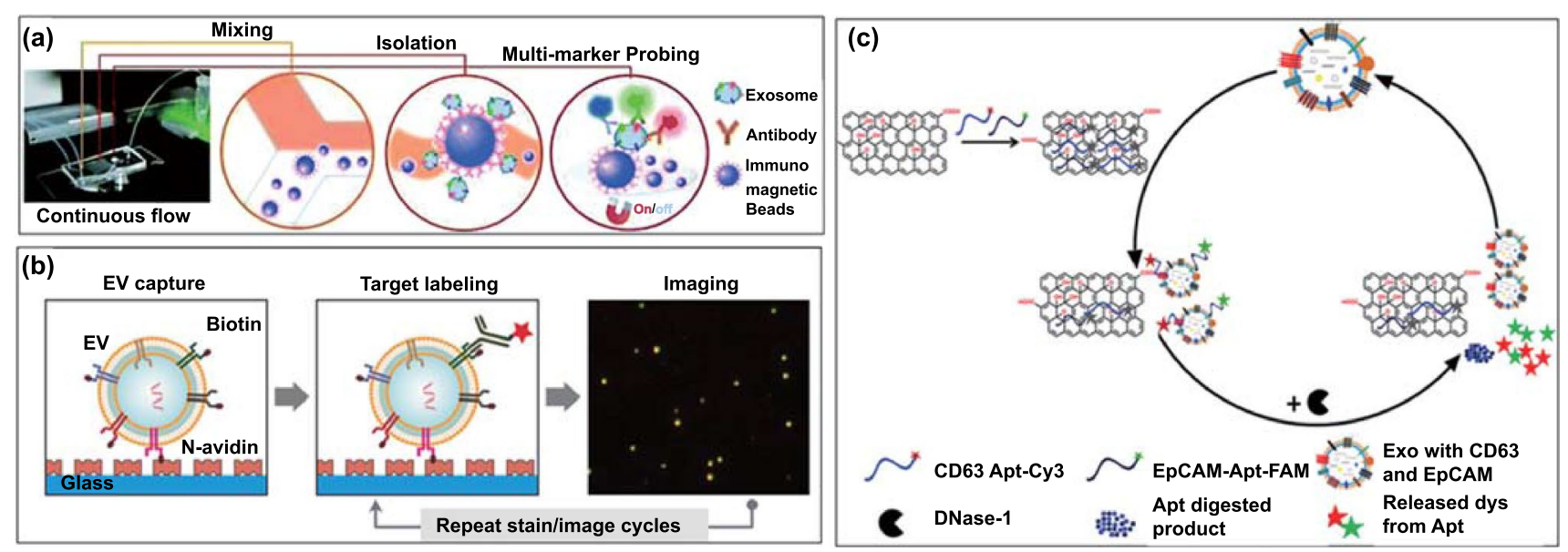

Fig. 4 Multiplexed profiling of EV proteins using fluorescent dye-based chemical coding strategy. a The ExoSearch chip for continuous mixing, isolation and in situ, multiplexed detection of circulating exosomal markers CA-125, EpCAM and CD24. Reproduced with permission from Ref. [43]. Copyright 2016 Royal Society of Chemistry. b Multiplexed single-EV analysis by microfluidic immunofluorescence staining. Reproduced with permission from Ref. [51]. Copyright 2018 American Chemical Society. c The principle of an enzyme-aided fluorescence amplification based on GO-aptamer interactions for the detection of exosomal membrane proteins. Reproduced with permission from Ref. [53]. Copyright 2018 Elsevier

were immunostained using different fluorophore-conjugated antibodies targeting different EV membrane proteins, followed by fluorescence imaging. Their measurement of 11 different proteins in a single vesicle uncovered a heterogeneous marker expression profile on individual EVs derived from the same glioblastoma cell line. For example, the results revealed that only half of the EVs are CD63 positive (54\%) and even fewer EVs expressed CD81 (26\%) and CD9 (4.8\%). In a different approach, Cavallaro et al., developed a multiple tagging strategy combining fluorescence imaging with atomic force microscopy which permits the acquirement of multidimensional information including membrane protein phenotype, size and mechanical properties simultaneously [52].

The multiplexed fluorescence profiling of EVs has been further extended to in situ liquid-phase detection using nanomaterial platforms. Want et al., for example, have explored a graphene oxide (GO) and fluorescent labelled DNA aptamers based platform for the detection of CD63 and EpCAM in colorectal cancer exosomes (Fig. 4c) [53]. Specifically, the fluorescence of fluorophore-labelled aptamers (Cy3CD63 and FAM-EpCAM aptamers) was quenched by GO but recovered by exosome induced displacement. The DNase I enzyme was then used to digest the single-stranded DNA aptamers on the exosome surface allowing the exosomes to interact with more fluorescent aptamer probes, resulting in further increase in the fluorescence signal. Such "switchon" and enhancement fluorescence profiling was shown to distinguish healthy from colorectal cancer patient samples using either CD63 or EpCAM. A similarly dispersed nanomaterial based approach was developed using GO with a panel of aggregation-induced emission luminogens (AIEgens) conjugated aptamers [54], or carbon nanotubes [55] or MXene $\mathrm{Ti}_{3} \mathrm{C}_{2}$ nanosheet [56] with different fluorescent dye labelled aptamers.

An amplified fluorescence multiplexing methodology was also reported using a thermophoretic aptasensor (TAS) for the enrichment of EVs and the profiling of seven surface proteins (PTK7, LZH8, HER2, PSA, CA125, EpCAM and CD63) via a panel of corresponding fluorescent aptamers (Fig. 5a) [57]. The EV membrane proteins were first labelled with fluorophore conjugated aptamers, followed by laser irradiation to generate a local temperature gradient to induce a size-dependent thermophoretic accumulation of EVs larger than $30 \mathrm{~nm}$. This led to a significant amplification of the fluorescent signal from aptamer bound EVs, a signal that linearly correlated with the respective protein expression levels [58]. The TAS was applied to 232 clinical serum samples from breast, liver, lung, lymph, ovary, and prostate cancer patients and healthy donors. A supervised machine learning algorithm was subsequently employed to classify different cancer types with an overall accuracy of $\sim 70 \%$ 


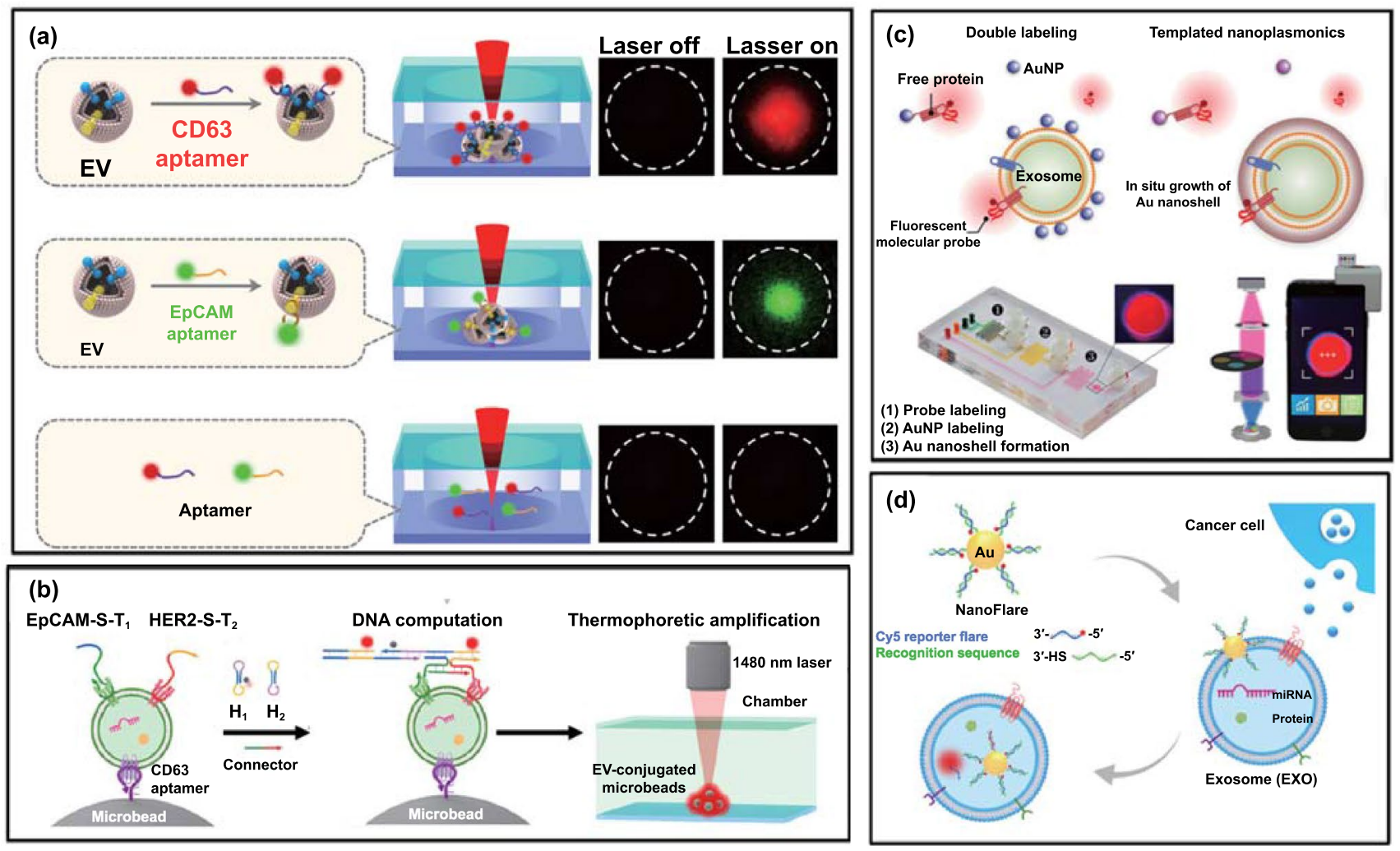

Fig. 5 Chemical coding strategies with signal amplification for EV multiplexing. a An aptasensor for the thermophoretic enrichment of EVs and multiplexed profiling of their surface proteins. Reproduced with permission from Ref. [57]. Copyright 2019 Springer Nature. b DNA ligation system for EV membrane protein profiling using thermophoresis. Reproduced with permission from Ref. [69]. Copyright 2021 American Chemical Society. c Schematic of the TPEX microfluidic multiplexing platform. Exosomes were incubated with different fluorescent aptamers, either individually (singleplex) or as a mixture (multiplex), for templated plasmonics for exosome (TPEX) analysis. Reproduced with permission from Ref. [63]. Copyright 2020 American Association for the Advancement of Science (AAAS). d Thermophoretic sensor implemented with nanoflares for in situ detection of exosomal miRNAs. Reproduced with permission from Ref. [70]. Copyright 2020 American Chemical Society

(Table 1). Moreover, a logistic regression analysis using these seven EV markers exhibited a superior performance to the routinely used prostate specific antigen (PSA) blood test in distinguishing prostate cancer from benign prostate disease. Taking advantage of thermophoretic accumulation, the same group further introduced a hybridization chain reaction (HCR) to achieve dual amplification for profiling multiple protein biomarkers at a single-EV level (Fig. 5b). In this study, tumour-derived EVs were first captured by CD63-aptamer-modified microbeads followed by addition of two aptamers targeting the membrane proteins EpCAM and HER 2 on EVs. The HCR was initiated with the two hairpins ( $\mathrm{H} 1$ and $\mathrm{H} 2)$ and two connector sequences to facilitate logic operation. After HCR, a thermophoretic enrichment of EV-conjugated microbeads amplified the output signal sufficiently to discriminate HER2-positive, HER2-negative patients, and healthy controls based on the convergent thermophoresis-DNA HCR. The concept of logic gate, as utilized here, is highly dependent on multiple aptamers being in close proximity. This proximity ligation approach was additionally verified for the sensitive detection of tumour-derived exosomal PD-L1/CD63 [59], and exosomal CD63/EpCAM/ MUC1 using multiple fluorophore coded aptamers [60-62].

In a different strategy, Wu et al. have explored an exosome-templated nanoplasmonic platform for exosome multiparametric molecular profiling (Fig. 5c) [63]. Specifically, cancer cell line conditioned media containing exosomes and free proteins were first incubated with three different fluorescent aptamers against CD63, EpCAM and MUC1 on AuNPs ( 9.2 nm). While AuNPs remain monodisperse when associated with a protein corona, they tend to encapsulate exosomes through electrostatic interactions with the 


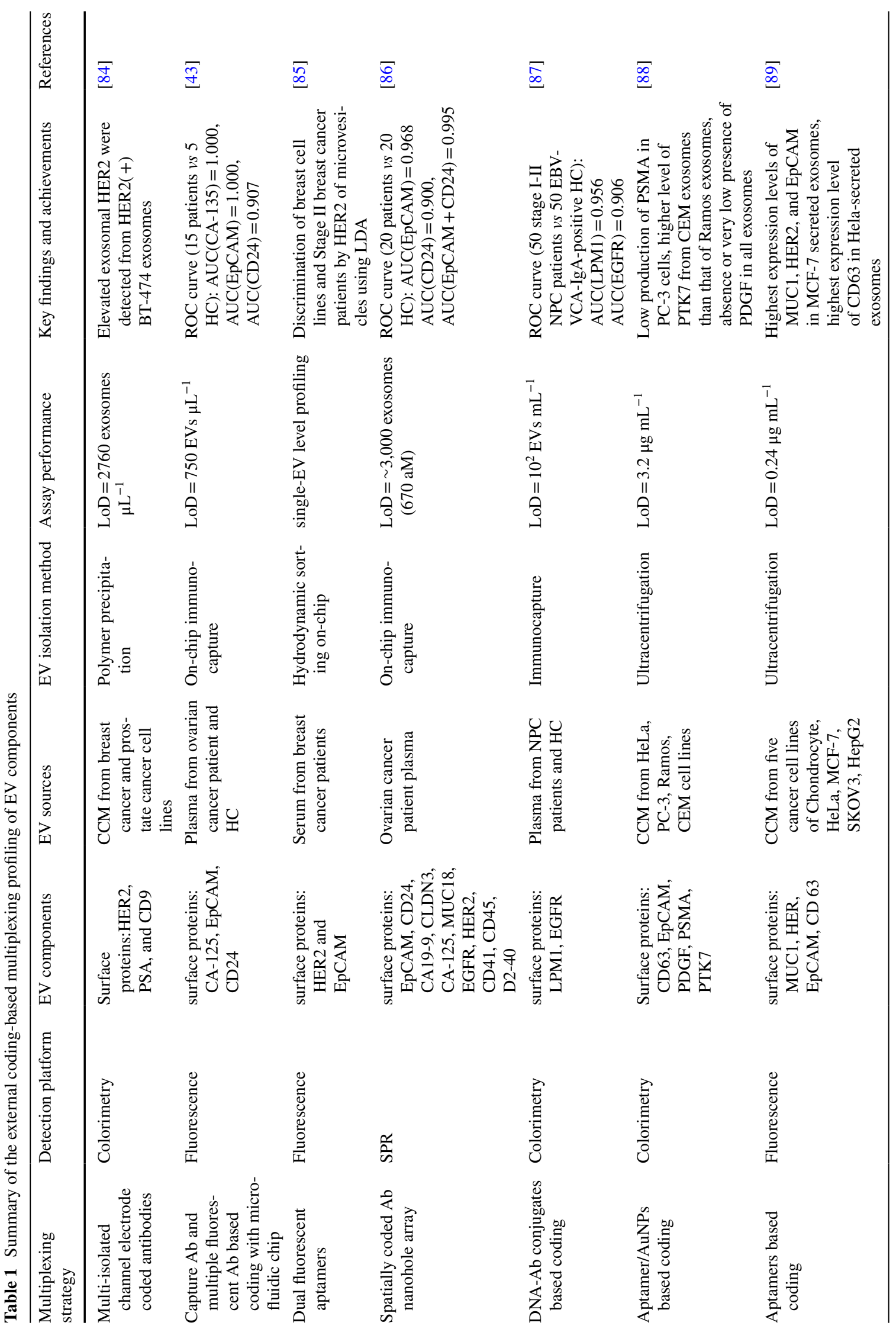




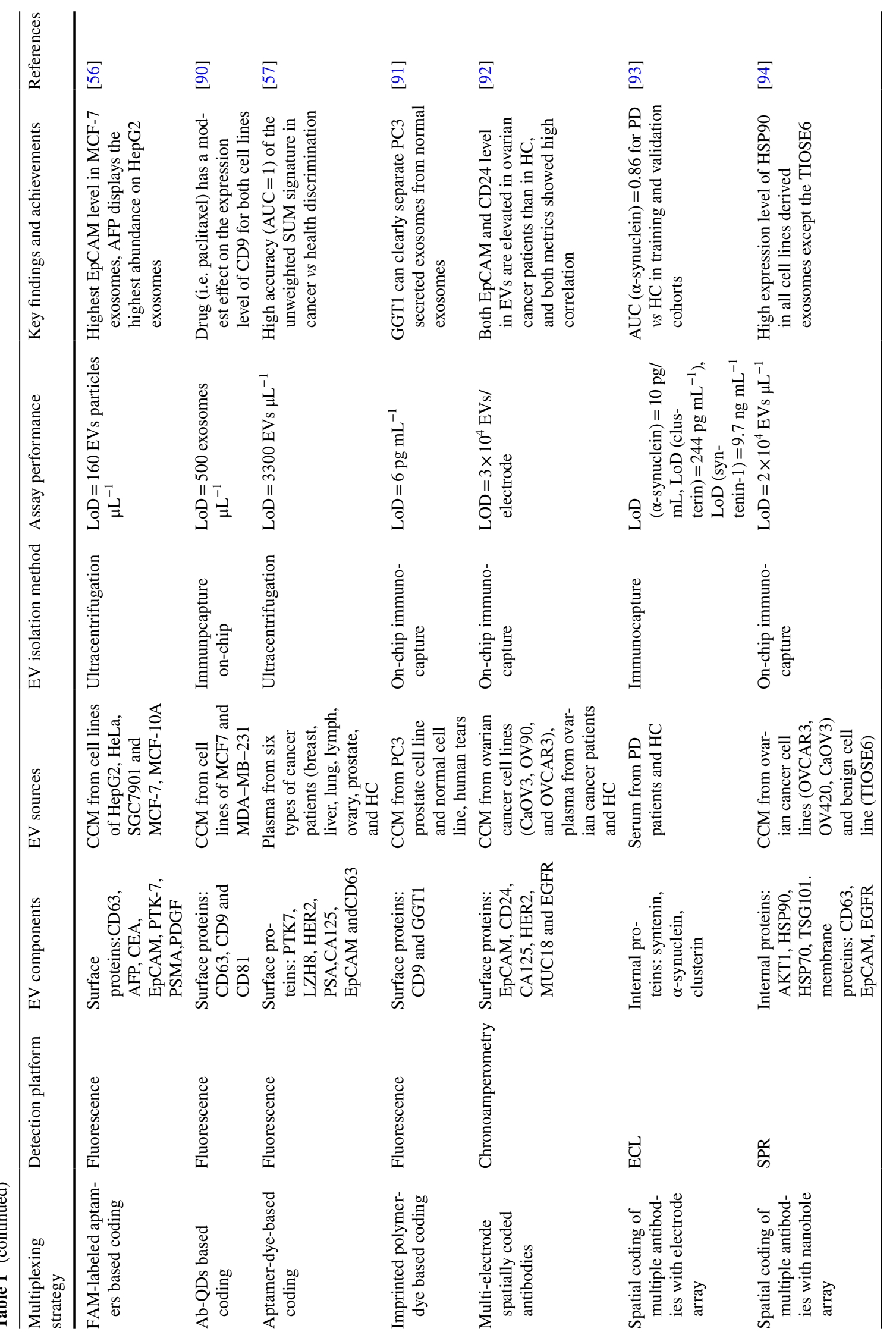




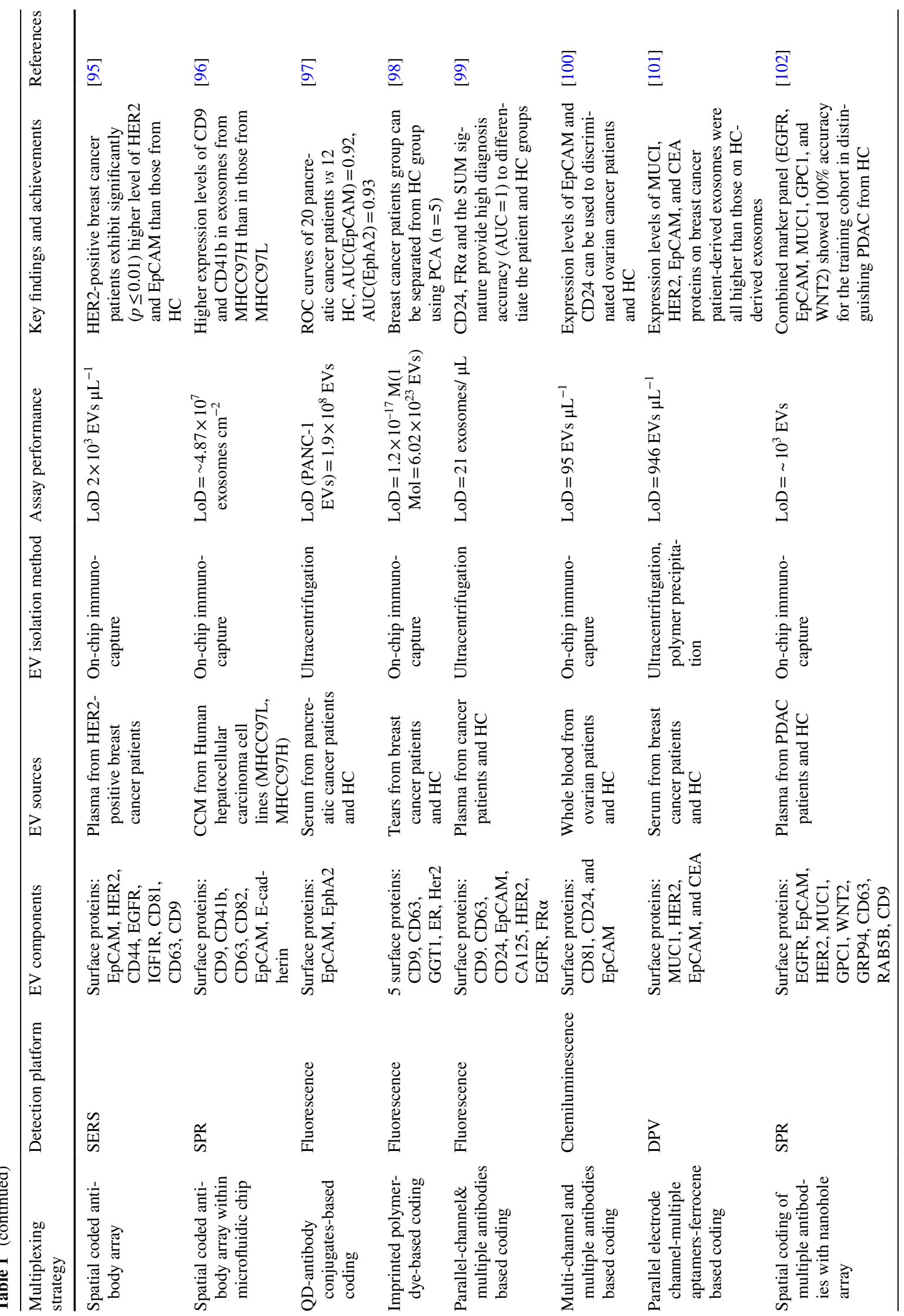




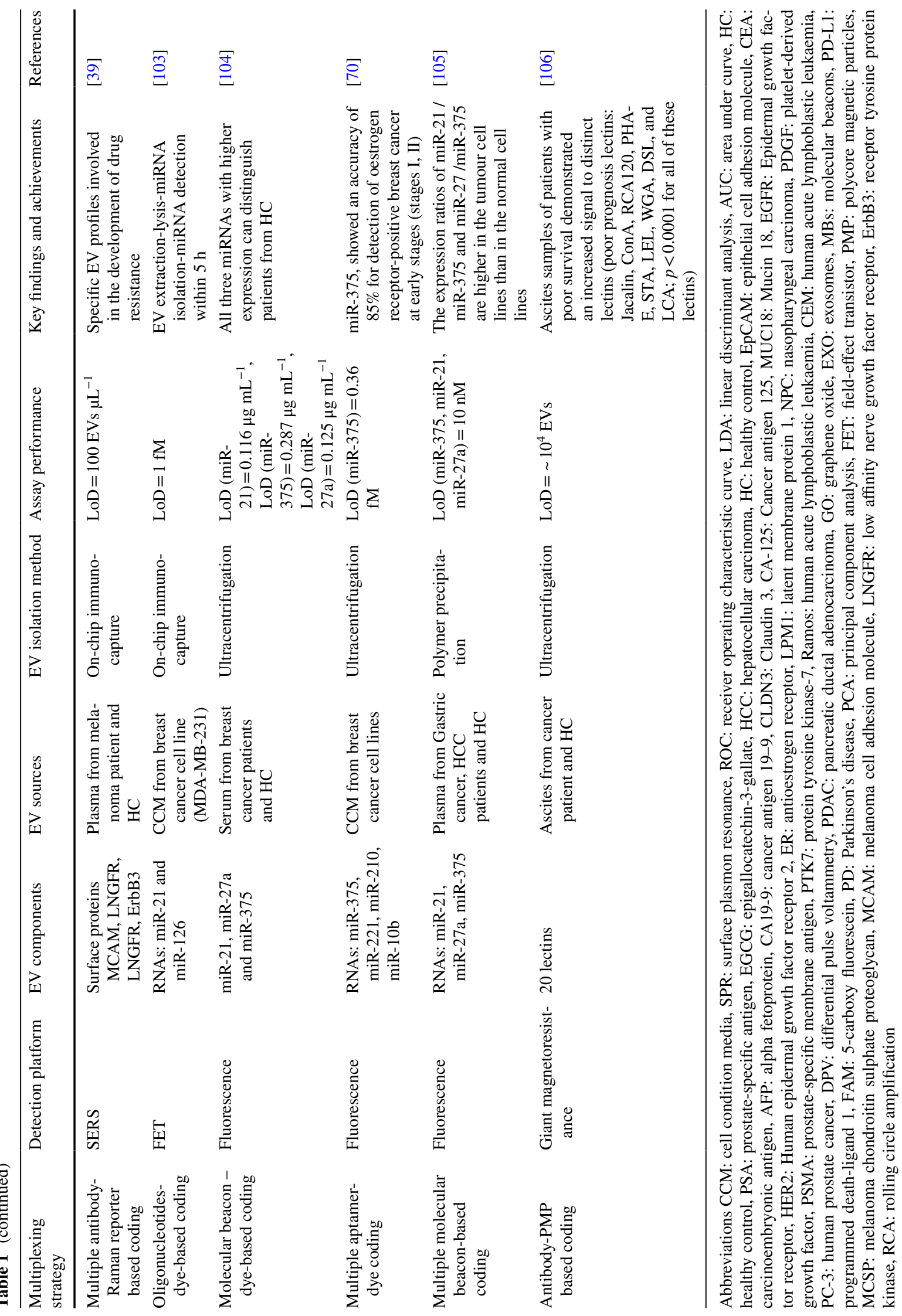


exosomal membrane. Excess unbound probes and AuNP are not removed. The AuNPs can then serve as seeds for in situ gold shell growth using exosomes as a template. Corona-coated AuNPs (or unbound AuNP) exhibit limited growth (and minimal red shift in their absorbance spectra), while AuNPs bound to the exosomal surface trigger strong localized plasmonic resonance in the infrared region. The nanoshell plasmonics locally quench the fluorescent probes only if both AuNPs and fluorophores bind on the same vesicle. The methodology thus supports an in situ analysis of EV surface proteins. The entire assay can be completed within 15 min using as little as $1 \mu \mathrm{L}$ of sample and additionally can be integrated with a smartphone readout. Further developments with the incorporation of more advanced microfluidics, such as droplet methods [64-67] and lithography patterned array [68], would enable highly parallel processing and facilitate large-scale clinical sample handling.

The examples discussed above concern fluorescent aptamers/antibody-based fluorescence coding for EV protein profiling. MicroRNAs (miRNAs) are also abundant in EVs. They are a class of small noncoding RNAs $\sim 22$ nucleotide long that regulate gene expression in a number of diseases, including cancer, neurodegenerative disorders, and diabetes [71] and as such they constitute a rich source of biomarkers. The main challenge in measuring exosomal miRNAs is their low abundance and instability [72].

Leveraging the versatility of thermophoretic profiling, exosomal RNA profiling can also be accomplished using RNA aptamers (Fig. 5d). To this end, Zhao et al. have introduced a nanoflare sensor comprising AuNPs (diameter of $\sim 13 \mathrm{~nm}$ ), 4 aptamers that recognise different miRNAs (targeting miR-375, miR-221, miR-210, and miR-10b), and Cy5 loaded complementary DNA probes [70]. Without contact with target miRNA, the fluorescence was quenched due to the close proximity of Cy5 to the surface of AuNPs. Upon passive uptake by exosomes, the reporter of nanoflares was displaced by the internal target miRNA, leading to an increase in fluorescence intensity. Under laser irradiation, the thermophoretic accumulation of nanoflare-treated exosomes leads to an amplified fluorescence signal upon the binding of exosomal miRNAs to nanoflares, allowing the direct measurement of exosomal miRNAs down to $0.36 \mathrm{fM}$ in $0.5 \mu \mathrm{L}$ serum samples. In this study, exosomal miR-375 showed an accuracy of $85 \%$ in detecting oestrogen receptorpositive breast cancer at early stages (stages I, II). Although this work offers a tool for EV molecular phenotyping, the requirement for an external laser source, and the potential associated damage to vesicles, requires further investigation before its translation into a point-of-care (PoC) device $[70,73]$.

Lee et al. developed the first molecular beacon-based assay for multiplexed detection of EV miRNAs [74]. Molecular beacons are hairpin-shaped oligonucleotide hybridization probes with a 3' fluorescent dye and 5' quencher. They serve as receptors that become fluorescent upon hybridization to a complementary RNA or DNA target sequence [75]. EV miRNAs consisting of miR-21, miR-375, and miR-27 derived from a breast cancer cell line of MCF-7 were studied (Fig. 6a). Using this approach, triple molecular beacons with unique fluorophores (FAM, Cy3, and Cy5) were successfully hybridize to three EV mRNAs (miR-21, miR-375, and miR27) within $1 \mathrm{~h}$, detecting in a single-step assay these markers in a dose-dependent manner. This in situ EV miRNA detection method avoids the need for EV lysis and RNA extraction, thus reducing the risk of RNA degradation.

To profile both EV proteins and miRNA within one configuration, Rhee and co-workers have explored the combination of fluorescent antibodies and molecular beacons for targeting membrane proteins and internal miRNAs, respectively [76]. As a proof of concept, Alexa Fluor ${ }^{\circledR} 488$-conjugated anti-CD63 antibody and molecular beacon-21 targeting miR-21 were investigated for multiplexed biomarker detection in healthy vs cancer-derived EVs using flow cytometry (Fig. 6b). To overcome the low resolution of flow cytometry, a phospholipid-polymer-phospholipid conjugate (DSPE-PEG-DSPE) was used to induce EV clustering due to the lipid solubility of DSPE. In a more recent study, Zhou et al. proposed a vesicle membrane infusion-based strategy to obtain multi-omics information of EVs [77]. As shown in Fig. 6c, anti-CD63 polystyrene beads were used to capture exosomes in a microfluidic channel, followed by fusion with fusogenic vesicles preloaded with three molecular beacons and three antibody-conjugated quantum dots (QDs) allowing the detection of 3 internal miRNAs (miRNA-451a, miRNA21, miRNA-10b) and 3 surface proteins (CD81, ephrin typeA receptor 2, carbohydrate antigen 19-9) through infusioninduced hybridization and immunoaffinity, respectively. The feasibility of infusion-hybridization strategy has also been extended to liposomes or cationic lipoplex nanoparticles for EV miRNA profiling in lung cancer [78-81], and human liver cancer [82]. These molecular beacon-based miRNA profiling methods are highly dependent on the integration 

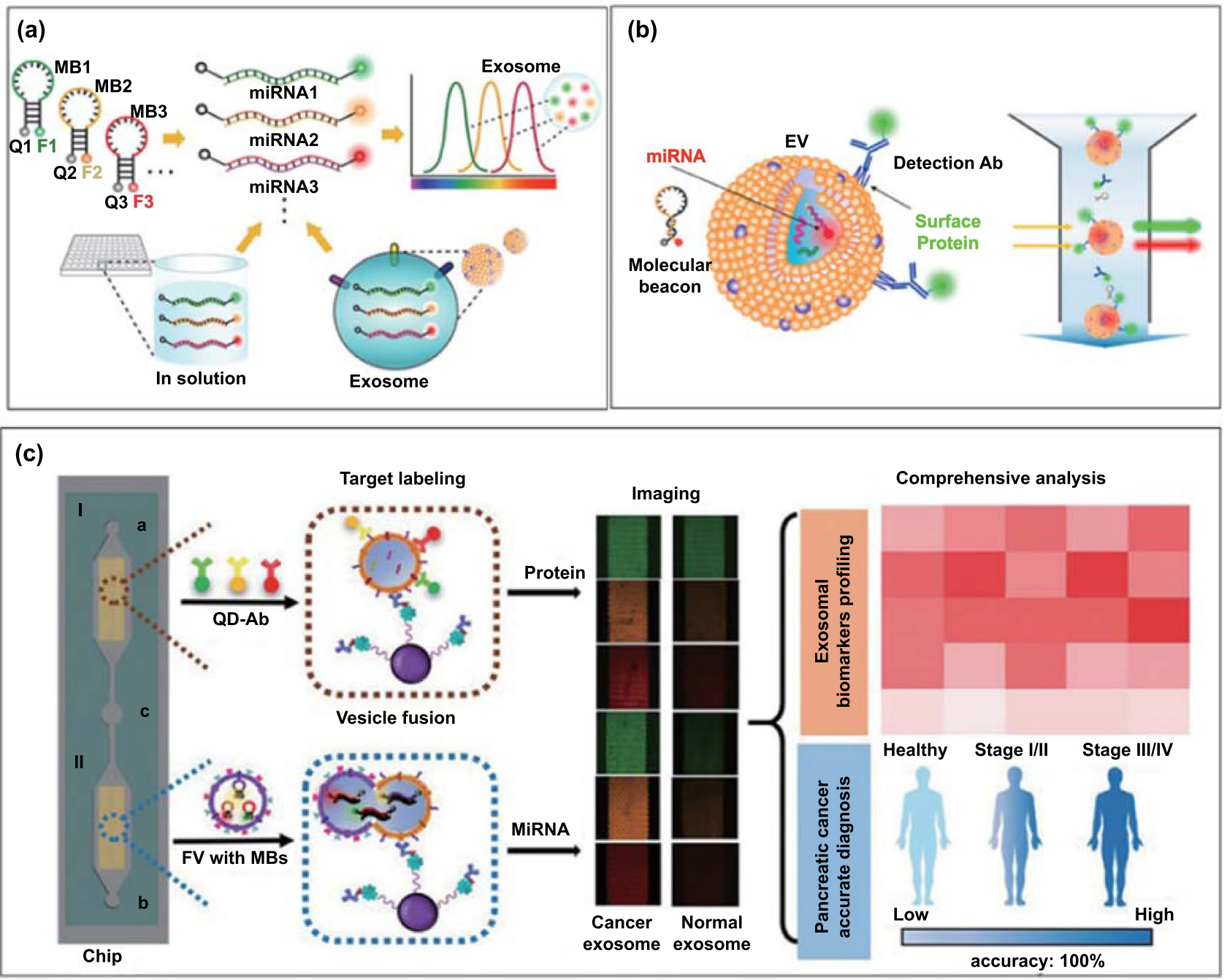

Fig. 6 a Molecular beacon-based exosome internal RNA triplexing ( $F$ : fluorescent dye. $Q$ : quencher). Reproduced with permission from Ref. [74]. Copyright 2016 Elsevier. b Simultaneous in situ detection of EV membrane protein and internal miRNA using dye conjugated molecular beacons and dye conjugated antibodies, respectively. Reproduced with permission from Ref. [76]. Copyright 2021 MDPI. c Simultaneous in situ detection of exosomal protein markers (CD81, ephrin type-A receptor 2, carbohydrate antigen 19-9) and miRNAs (miRNA-451a, miRNA-21, miRNA-10b) using QDs labelled antibody and molecular beacons using fusogenic vesicles in a microfluidic device. Reproduced with permission from Ref. [77]. Copyright 2020 John Wiley \& Sons

with fluorescence microscopes; a translation to a scalable or PoC setting, mobile phones coupled with near-infrared fluorescence imaging [83] might be applied.

In summary, a number of approaches have been employed to detect multiple EV analytes (Table 1). The optical/electric/electrochemical multiplexing strategies for proteins can be readily adapted for RNAs by replacing the relevant receptors. However, fundamental issues remain: (1) the correlation between lysed EV RNA and nonlysed in situ EV RNA detection using the same platform to evaluate the uptake and capture efficiencies, (2) exploration of more generic signal amplification approaches especially when thermophoresis is not applicable. (3) Although fluorophores with different excitations can be employed for simultaneous detection, the potential for scale-up multiplexing remains due to the limited choice of chemical reporters, spectrum overlap, nonspecific adsorption and cross-talk in close proximity. For example, the overlap between the emission spectrum of FAM and the excitation 
(a)
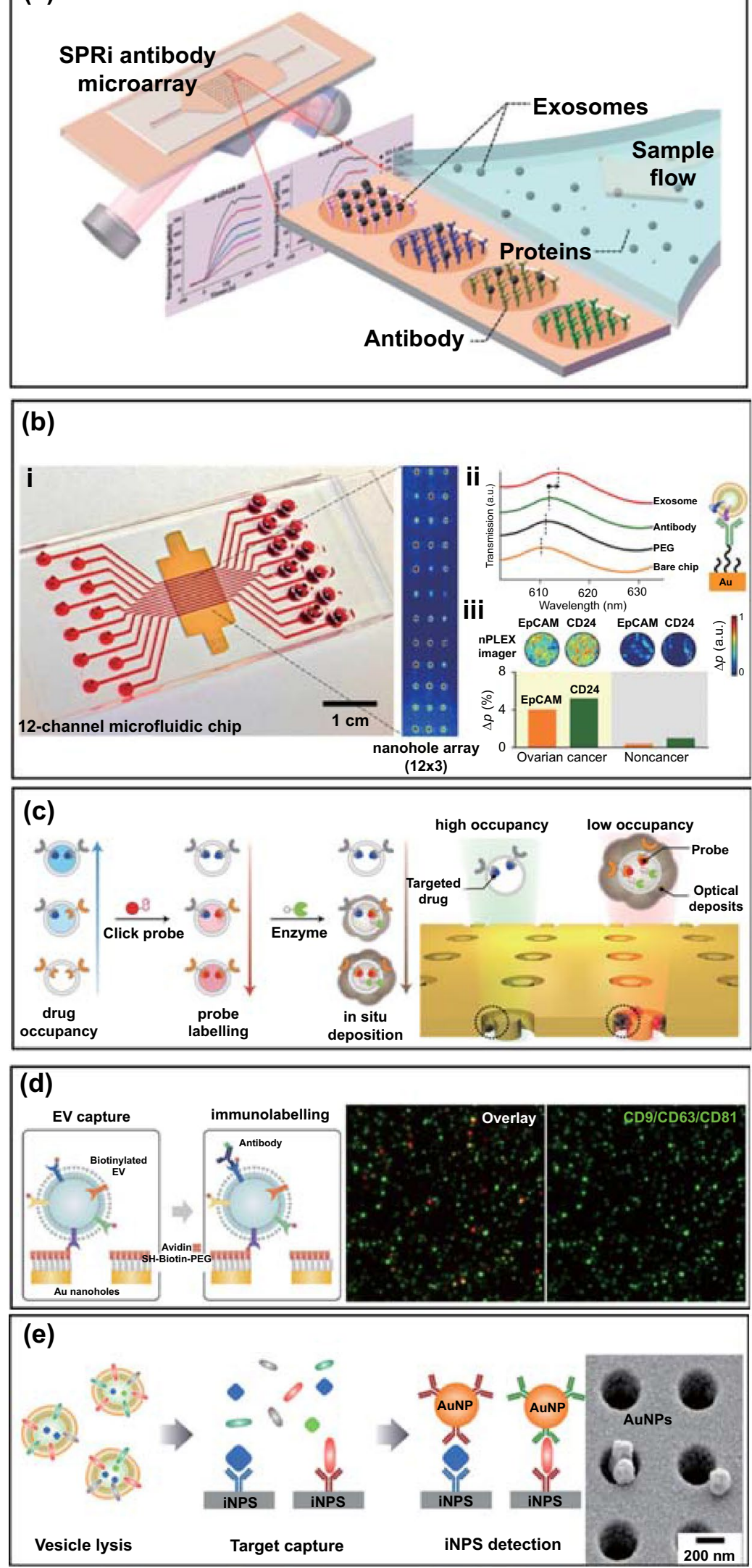
\Fig.7 Schematic view of a physical spatial coding-based SPR platform for EV multiplexing. a Antibodies specific to EV transmembrane proteins were printed on the gilded gold chip, and integrated into a flow cell. Reproduced with permission from Ref. [96]. Copyright 2014 American Chemical Society. b nPLEX chip. (i) Integration of a multi-channel microfluidic cell for independent and parallel analyses. Transmission intensities of $12 \times 3$ nanohole arrays were measured simultaneously using the imaging setup. (ii) A representative schematic of changes in transmission spectra showing EV detection with nPLEX. (iii) Ascites-derived exosomes from ovarian cancer and noncancer patients were evaluated by the nPLEX sensor. Cancer EVs were captured on EpCAM and CD24-specific sensor sites with associated intensity changes in the transmitted light. Adapted with permission from Ref. [86]. Copyright 2014 Springer Nature. c Enzymatic amplified plasmonic sensor for EV multiplexing. Reproduced with permission from Ref. [117]. Copyright 2019 Springer Nature. d Surface plasmon-enhanced fluorescence biosensing for EV multiplexed profiling. Reproduced with permission from Ref. [118]. Copyright 2020 John Wiley and Sons. e Intravesicular nanoplasmonic system for EV multiplexing. Reproduced with permission from Ref. [94]. Copyright 2018 American Chemical Society

spectrum of Cy5 is too small to cause Förster resonance energy transfer (FRET).

\section{Physical Spatial Coding}

Physical spatial coding is another widely explored strategy for EV multiplexing, typically realized by positioning different receptors on an array or separated chambers targeting EV components at different spots in an addressable way (e.g. position A1, B1 represent protein A and B, respectively). The binding events of EV analytes to the receptors can be monitored using either surface sensitive label-free methods (e.g. surface plasmon resonance, SPR, quartz crystal microbalance, QCM, electrochemical impedance spectroscopy, EIS) or external chemical tags in a convergent physico-chemical coding fashion.

\subsection{SPR Arrays}

The surface plasmon resonance (SPR) sensing platform is exceptionally versatile for probing interfacial molecular binding events through an evaluation of the refractive index change and has been intensively investigated for highly sensitive detection of EVs [107-110]. With the integration of a charge-coupled device (CCD) camera in a spatially resolved imaging mode (SPRi), it can analyse multiple simultaneous binding events. EV multiplexed assays using an SPR sensor can usually be realized with multi-spot patterned arrays, which are spatially encoded by printing or lithography, coated with multiple EV receptors (e.g. antibodies) targeting specific EV components. In a typical example, Zhu et al. designed an SPR chip with an antibody microarray to simultaneously quantify multiple EV transmembrane proteins (Fig. 7a) [96]. The gold surface was printed with antibodies using a commercial microarray printer and was integrated into a microfluidic chip. Upon injection of the sample into the flow cell, EV were captured, and the associated refractive index change was monitored by the CCD camera. The SPR array has been used to detect the expression of six membrane proteins (e.g. CD9, CD41b, CD63, CD82, EpCAM, E-cadherin) on intact EV harvested from human hepatocellular carcinoma cells. The authors demonstrated that the expression of EV CD9 and CD41b was reduced after Rab27a-siRNA (i.e. small interfering RNA) treatment and increased after monensin treatment. These results demonstrated the potential of using antibody-coated microarraybased SPRi biosensors in cancer detection and drug treatment evaluation (Fig. 1c) [111].

To increase throughput capacity, Im et al. developed a plasmonic multiplexing EV platform (i.e. nPLEX) by integrating a $12 \times 3$ periodical nanohole gold array-based transmission SPR chip into a 12-channel PDMS chip (Fig. 7b) [86]. The chip surface was modified with mixed PEGbased SAMs (1:3, longer biotin- $\mathrm{PEG}_{1000}$-SH and shorter $\left.\mathrm{CH}_{3}-\mathrm{PEG}_{200}-\mathrm{SH}\right)$ to allow antibody conjugation while resisting nonspecific fouling $[112,113]$. This enabled the detection of twelve different $\mathrm{EV}$ membrane proteins using antibodies. Signals were further amplified by attaching anti-CD63-modified gold nanostars to captured EVs and utilizing the plasmonic coupling of these to the underlying gold surface [114-116]. The authors showed the clinical potential of this approach using ascitic fluid from 20 patients with ovarian cancer and 20 controls.

The periodic nanohole SPR array has also been employed recently to evaluate pharmacodynamics based on an examination of EV membrane protein expression after drug treatment [117]. As presented in Fig. 7c, four bio-orthogonal click probes modified with trans-cyclooctene (TCO) were designed to enable the specific targeting of three cancer proteins (EGFR, EpCAM and MUC1) and one generic EV marker (CD63). In this mode, lower drug occupancy on the EV membrane protein site allows higher orthogonal probes 

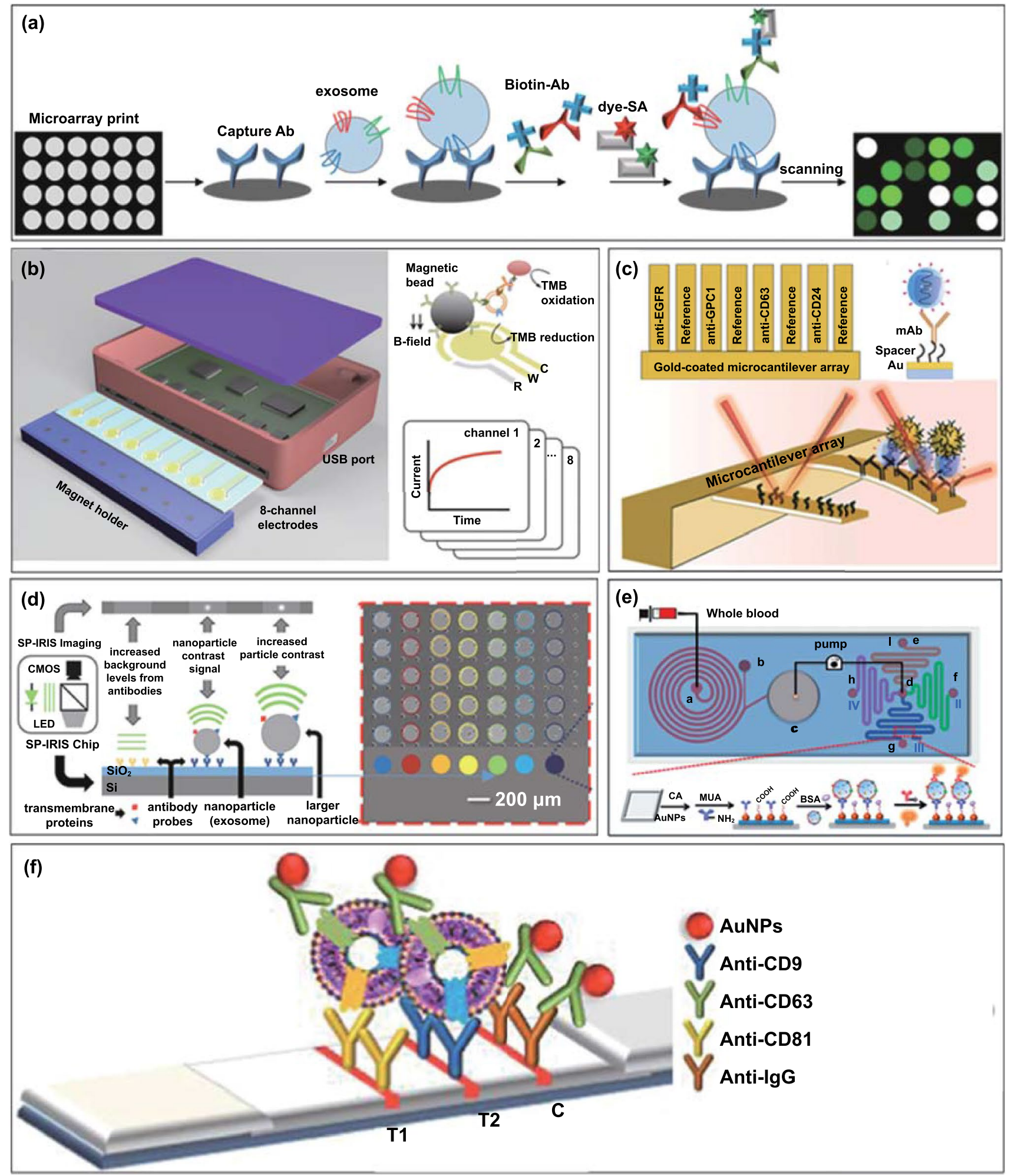

to engage the receptor site, leading to more enzyme (horseradish peroxidase, HRP) recruitment and in situ conversion of the soluble substrate (3,3'-diaminobenzidine) into insoluble product causing a spectral shift. This signal can be amplified due to the confined nanoring cavities where strong electromagnetic hotspots reside, so supporting a 
4Fig. 8 Non-SPR physical coding-based multiplexed profiling of EVs. a Schematic view of EV array detection of EV proteins. Adapted with permission from Ref. [123]. Copyright 2013 Taylor and Francis. b Integrated magnetic-electrochemical exosome (iMEX) platform. The sensor can simultaneously measure signals from eight parallel electrodes. Reproduced with permission from Ref. [92]. Copyright 2016 American Chemical Society. c Antibody modified cantilevers in the array with a reference control for differential detection of signal (up). Schematic of the effect of the nanoparticle mass loading on the nanomechanical deflection of the cantilever (down). Adapted with permission from Ref. [140]. Copyright 2016 Royal Society of Chemistry. d Schematic representation of the Single Particle Interferometric Reflectance Imaging Sensor (SP-IRIS) detection process. SP-IRIS detection principle, monochromatic LED light illuminates the sensor surface and the interferometrically enhanced nanoparticle scattering signature is captured on a CMOS camera (left). Low-magnification interferometric image showing microarray of immobilized capture probes (right). Reproduced with permission from Ref. [141]. Copyright 2016 Springer Nature. e Schematic diagram of an integrated microfluidic chip for plasma separation, EV detection, and molecular analysis. Reproduced with permission from Ref. [100]. Copyright 2020 American Chemical Society. f Multi-test line strip for profiling of EV membrane proteins. Reproduced with permission from Ref. [142]. Copyright 2017 Elsevier

sensitive ability to monitor changes in protein composition and drug occupancy. The proposed platform was demonstrated to identify disease status and rapidly $(\sim 1 \mathrm{~h})$ distinguish between treatment outcomes using as little as $5 \mu \mathrm{L}$ of plasma. In another recent study, Min et al. explored long-range (>100 nm) SPR-enhanced fluorescence on a periodic array instead of the localized "hotspot" surfaces $(<20 \mathrm{~nm})$. This approach is more suitable for measurements with intact EVs given their dimension (30-1000 nm) $[118,119]$. Four fluorescent dyes (AF488, Cy3, Cy5, and Cy5.5) were conjugated with streptavidin separately in order to code biotinylated EV membrane proteins (CD9, CD63, CD81, GAPDH, EGFR, EGFRvIII) after their capture on neutravidin-PEG layers (Fig. 7d). The PEG layer was used to prevent fluorescence quenching and to resist nonspecific adsorption [120]. This work presents a typical combination of physical and chemical coding approach powered by plasmon enhanced fluorescence $(\sim 10 \times)$.

Park et al. have extended the nanoplasmonic array $(10 \times 10$ array) platform to measure both internal (AKT1, HSP90, HSP70, TSG101) and transmembrane proteins (CD63, EpCAM, EGFR) in lysed EVs derived from ovarian cancer cells (Fig. 7e), coupled with induced localized amplified signals generated by immunogold labelling [94] . By lysing EVs, this approach provides one way to overcome the compromise of signal enhancement in the confined "hot spot" design (i.e. detection antibody conjugated AuNPs-EV and analyte-capture antibody modified gold interface) due to steric hindrance and the dimension of intact EVs. The authors demonstrated that their sensor enabled $>10^{2}$-fold higher sensitivity with reduced sample input $(0.5$ vs. $100 \mu \mathrm{L})$ when compared to standard ELISA. Increased expression of HSP90 and HSP70 and decreased levels of AKT1 and TSG101 were observed in cancer cells treated with a model drug, suggesting that drug-dependent EV protein signatures may be applied to monitor therapies in patients.

In summary, the binding events of EV components to the sensing interface can be monitored by surface sensitive techniques such as SPR in a label-free format or by labelling them with one or multiple reporters, i.e. using a combination of physico-chemical coding strategies [118]. The advanced nano-/micro-fabrication-assisted SPR arrays and their integration into microfluidic chambers have been increasingly employed to facilitate better sample manipulation and miniaturization. Nanomaterial-based signal amplification approaches $[110,121,122]$ can be applied to further improve the sensitivity of so developed EV sensor arrays.

\subsection{Other Spatial Coding Platforms}

\subsubsection{Antibody Arrays}

Antibody array designed high-throughput multiplexing platforms, based on the immobilization of antibodies on the surface of microfluidic biochips, have been extensively applied to the analysis of EV biomarkers [123, 124]. In such configurations, an immune-based isolation of EVs is followed by a target specific immunofluorescence step. Where a panel of antibodies is spatially coded on a biochip surface, such analyses are multiplexed [125]. For example, Jørgensen et al. developed an EV microarray capable of detecting up to $60 \mathrm{EV}$ proteins from $10 \mu \mathrm{L}$ plasma (Fig. 8a) [123]. A panel of 60 captured antibodies were printed on epoxy-coated slides to recognize generic EV markers (e.g. CD9, CD63) or tumour-associated markers (e.g. EpCAM, HER2, p53). Plasma-derived EVs were captured on slides, then tagged with biotinylated anti-CD9, CD63, and CD81 antibodies prior to a readout with fluorescent streptavidin (down to detection limits of $2.5 \times 10^{4}$ EVs per detection spot). 


\subsubsection{Multi-channel Electrochemical Sensors}

Electrochemical detection represents another promising platform for multiplexed $\mathrm{EV}$ analysis owing to its intrinsic high detection sensitivity and compactness and potentially automated setup [111, 126]. Electrochemical multiplexed profiling can usually be realized by integration of multiple receptors with multi-channel electrodes (i.e. spatial position coding) and/or by cooperation with different redox probes labelled receptors, to detect the current changes as a function of the EV biomarker levels [127, 128].

For example, Jeong et al. developed a portable integrated magneto-electrochemical EV biosensor (iMEX) for multiplexed EV protein detection (Fig. 8b) [92]. The iMEX biosensor used a multi-channel (i.e. eight parallel electrodes) design offering simultaneous detection of eight markers within $1 \mathrm{~h}$, with each marker requiring only $10 \mu \mathrm{L}$ of plasma. In this work, magnetic immunobeads coated with anti-CD63 antibodies were used to enrich for CD63 positive EVs, followed by detection with antibodies against six surface proteins (see Table 1 for markers) conjugated to horseradish peroxidase (HRP) loaded magnetic beads (a chronoamperometric signal being generated). The LoD of the iMEX biosensor was $3 \times 10^{4} \mathrm{EVs}$ per electrode, outperforming conventional ELISA by 100-fold higher sensitivity. The same group have also reported another magnetoelectrochemical (multiple-electrode) sensor, termed iKEA (i.e. integrated kidney exosome analysis), to detect kidney transplant rejection. The device enabled a fast screening of $\mathrm{T}$ cell-derived EVs for lymphocyte-specific protein detection (CD3, CD45, CD68, CD2, HLA-ABC, CD52), showing that high levels of CD3-positive EVs identify kidney transplant rejection patients with in $91.1 \%$ of cases tested [129]. A further scale-up of this approach was reported by Tang et al., who explored an electrochemical array featuring 32 individually addressable microelectrodes multiplexed with an 8-port manifold to provide 256 sensors [130] that was successfully used to detect prostate cancer proteins in serum.

An electrode array modified with multiple receptors has also been employed for the multiplexed detection of EV miRNAs. Goda et al. have specifically reported the parallel detection of EV microRNAs (miR-143 and miR-146a) using a microelectrode array by potentiometry after reverse transcription polymerase chain reaction (RT-PCR) on EV lysates [131]. This sensing interface is based on DNA functionalized, mixed self-assembled monolayer (SAM) electrodes, comprising 5-SH- $\left(\mathrm{CH}_{2}\right)_{6}$-DNA probe and zwitterionic sulfobetaine (SB) units as antifouling agents [113, 132]. Target hybridization events lead to changes in the interface potential which are transformed into a potentiometric signal. The platform was applied to a duplex assessment of miR-143 and miR-146a [133, 134] achieving an LOD of $\sim 20 \mathrm{pM}$. Although such a platform is multi-step in nature (EV isolation-lysis-RT-PCR-detection), it is potentially readily integrated into microfluidic pre-steps and the utilized surface chemistry could be implemented in other EV analysis platforms $[131,135,136]$.

\subsubsection{Multi-channel Nanomechanical Sensor}

Spatial coding-based EV multiplexing is also compatible with other surface sensitive detection techniques. In this regard, mechanical biosensors, e.g. microcantilevers, suspended micro-and nanochannel resonators, and quartz crystal monitoring have been utilized in bioanalysis where they can exhibit both high sensitivity and fast response [137, 138]. For example, Olcum et al. previously measured the mass of individual EVs by using a suspended nanochannel resonator (SNR) system with the ability to weigh individual nanoparticles at attogram scales [139]. Inspired by this method, Etayash et al. reported a nanomechanical cantilever array system for the simultaneous detection of multiple EV surface antigens (Fig. 8c) [140]. This nanomechanical system consisted of a gold-coated microcantilever array, on which specific EV-capturing antibodies were separately integrated. The so-generated cantilever defections generate detectable mechanical signals that scale with specific EV concentration. The use of antibody (anti-Glypican-1)-modified AuNPs to recognise and bind to pre-captured EVs further improves detection sensitivity down to $\sim 200 \mathrm{EV} \mathrm{mL}^{-1}$. Despite these advances, the scalable fabrication and modification of nanomechanical biosensors remain technically challenging.

\subsubsection{Interferometric Reflectance Arrays}

Other label-free optical methods, such as interferometric reflectance imaging sensors (IRIS) have been used for multiplexed EV assessments. The IRIS technology is based on enhanced contrast in a scattering signal from captured nanoparticles generated on a layered substrate. This technology 
is widely applied to the simultaneous detection of multiple viruses in human blood [143]. Daaboul and co-workers have, for example, recently developed a surface-sensitive interferometric reflectance imaging sensors (IRIS) for the multiplexed phenotyping and digital counting of subpopulations of individual EVs captured on a microarray (Fig. 8d) using antibodies against the standard surface markers CD9, CD63 and CD81 [141]. The contrast generated is sufficient for single-EV counting with spatial location in the array encoding phenotype information. Such platforms can be readily extended to include additional EV membrane proteins by coating with corresponding receptors on different surface locations.

\subsubsection{Multi-channel Microfluidics}

Liu et al. designed a spinal microfluidic chip integrated with an EV detection module, enabling the direct separation and detection of EVs derived from a human ovarian cancer cell line and also plasma (Fig. 8e) [100]. The separation chip consisted of six annular microchannels that separated the plasma under inertia, reducing the interference from cell fragments and contents. The supernatant obtained after separation entered a detection module consisting of four isolated S-shaped channels, modified with capture antibodies for IgG, CD24, CD81 and EpCAM, respectively. After EV capture, HRP-labelled detection antibodies were added for downstream chemiluminescent (CL) quantification of three membrane proteins. This sensory chip was used to monitor the clinical response of ovarian cancer patients $(n=11)$ after treatment with surgical resection or chemotherapy. Levels of both CD24 and EpCAM in EVs were shown to decrease among responding patients $(n=8,1-6$ directed surgical resection, 8 and 9 treated with chemotherapy), indicating that the proposed device could be used to monitor response to cancer therapies.

\subsubsection{Multi-test line Lateral Flow Strips}

The lateral flow immunoassay (LFA) strip is a widely used assay for routine testing due to the small sample input required and low cost. In this format, pre-embedded capture antibodies bind the target analyte (i.e. at a test line) with IgG used as quality control (e.g. control line). Upon addition of the sample, AuNP decorated detection antibodies bind to the target forming a sandwich immune-complex and so generate a colorimetric signal. Blanco-Lópeza et al., for example, have introduced an EV multiplexing strip by using three antibodies targeting the corresponding membrane proteins of CD9/CD61/CD81 (Fig. 8f). Different labels (AuNPs, carbon black and magnetic nanoparticles) were examined, and AuNP-modified detection antibodies were shown to enable a sensitivity of $8.54 \times 10^{5} \mathrm{EVs} \mu \mathrm{L}^{-1}$ using plasma samples [142]. Guo et al. have further explored the application of isotachophoresis in LFA in a paper-based isotachophoresis (ITP) format. As an electrokinetic sample-focusing technique, ITP requires a discontinuous electrolyte system comprising a leading electrolyte (LE) and a terminating electrolyte (TE) with the capability of concentrating samples by multiple orders (100-1000) of magnitude in minutes with minimal sample consumption [144]. Anti-CD44 and anti-CD63 antibodies were here used to capture cancer and generic EVs. This system performed well in human serum with target concentrations as low as $1.2-2.0 \times 10^{3} \mathrm{EVs}_{\mu \mathrm{L}}^{-1}$ [145].

As a highly versatile and adaptive detection platform, a wide range of engineering strategies like multiple labelling using nanomaterials, nucleic acid amplification and antifouling surface chemistry, can be employed in LFA-based $\mathrm{EV}$ analysis to enhance analytical performance in terms of sensitivity and selectivity [146-151]. Because of this, LFA is much cited PoC platform for EV analysis, but its utility is likely to be limited to EV surface proteins.

In summary, there is a wide range of non-SPR chip-based multiplexing platforms available, with a generally good capability of integration with functional modules like onchip EV capture and wash. Most studies to date have focused on surface EV marker profiling, with the internal protein or RNA content not often explored. The integration of a lysis unit together with an isolation-detection module could allow a more comprehensive exploration of EV markers and accelerate biomarker discovery.

\section{Biological Coding}

In biological coding-based EV multiplexing, the EV component of interest is labelled with nucleic acids, followed by detection of these sequences, e.g. using chromatography or PCR, the binding event of EV analytes is converted to the detection of DNA fingerprint-based molecular tags (often with 
an associated significant amplification). Capillary electrophoresis (CE) can achieve high separation resolution and detection sensitivity with low sample consumption and easy maintenance $[44,152,153]$. Under optimal conditions, multiple analytes can be separated in terms of different retention time and assessed using $\mathrm{CE}$ owing to their different molecular weights and charges [154]. Singh et al., for example, have explored CE coupled with polymerase chain reaction-amplified immunoassay (I-PCR) for the simultaneous detection of multiple surface proteins (CD9, CD34, CD63, CD123, c-Kit/CD117, FLT-3/CD135) in EVs from acute myeloid leukaemia (AML) (Fig. 9a) [155]. In this work, EVs were first captured by antiCD63 antibodies, followed by binding of different detection antibodies tagged with reporter DNA. Such a sandwich format combined the specificity of an immunological recognition with the exponential amplification of PCR. This method achieved the simultaneous detection of the five analytes within $12 \mathrm{~min}$ with a limit of detection (LOD) of $2 \mathrm{EVs} \mu \mathrm{L}^{-1}$ for leukaemia cell-derived EVs. A major limitation, however, concerns the poor mass separation resolution of $\mathrm{CE}$. This problem can be resolved by nongel sieving $\mathrm{CE}$ in which entangled hydroxypropylmethylcellulose (HPMC) polymer solutions can be used instead of cross-linked gels like agarose or polyacrylamide $[44,153]$. The concept of an integrated DNA amplificationcapillary electrophoresis microfluidic system could enable the development of portable devices for molecular typing of $\mathrm{EVs}$ in other complex diseases [154, 156]. Inspired by such idea, Ko et al. have recently reported the application of antibodyDNA conjugates to label EVs, followed by stochastic microfluidic incorporation of single EVs into droplets (Fig. 9b) [157]. This was followed by in situ PCR with fluorescent reporter probes which amplify the barcode signal for subsequent detection with droplet imaging. This immune-droplet digital polymerase chain reaction (iddPCR) amplification method enabled the multiplexed profiling of EV proteins, e.g. EGFR, EPCAM, PD-L1, CD4, CD8, GZMB, TCF7 derived from cancer cell lines. This is a typical example that incorporates different coding strategies (biological and chemical coding) with amplification, to enable the detection of multiple EV membrane proteins at single-EV resolution [158-160]. It should be noted, however, that the most common formats using antibody-DNA conjugates require multiple synthesis and purification steps and a careful assessment for false-negative or false positive results (Table 2).

\section{Nanoparticle Coding}

In addition to the above-discussed chemical-, physical- and biological-based EV multiplexing strategies, nanoparticlebased coding is a significant and growing option. Due to their easy synthesis and tuneable physiochemical properties, a broad range of nanoparticles bearing distinct properties (e.g. fluorescent-, plasmonic-, electrochemical-) have been used as labelling tags for the generation of different readouts in multiplex EV profiling. Nanoparticle coding, which can utilize numerous types of nanoparticles for signal generation to present EV analytes. This approach can be considered as an extension of the chemical tag-based coding approach.

\subsection{Fluorescent Barcoded Beads}

The combination of fluorescent barcoded beads with flow cytometry (FCM) is a widely applied detection approach. EVs are typically too small to be detected by FCM directly by conventional flow cytometers but an adaptation of the technique can be used to quantify multiple EV protein markers [161]. This can be generically achieved by two immune-sandwich formats, i.e. capture antibody modified microbeads-EVs-fluorescent detection antibodies [162] or capture antibody modified barcoded microbeads-EVsallophycocyanin (APC) or phycoerythrin (PE)-conjugated detection antibodies (Fig. 10a) [163-165]. When a laser beam illuminates EVs, the scattered light is converted to an intensity-associated voltage pulse that can be quantified. Depending on marker selection, the bead EV-based FCM allows the evaluation of different subpopulations in a single sample or the comparison of EV subpopulations in different samples. Koliha et al., for example, have described a multiplex bead-based FCM platform for the analysis of different subpopulations of EVs where up to $39 \mathrm{EV}$ surface proteins in different cell lines and subsets of rare cells were analysed in work demonstrating the strength of this method in profiling heterogeneous EV subpopulations [162].

A range of commercial analysis platforms are available from Becton Dickinson and Company (Cytometric Bead Array) and the partners of Luminex Corp (хМАРтм), with products for in vitro diagnostics (IVD). These utilise sandwich immunoassays consisting of beads that differ in size or fluorescence intensity (colour or scattering-coded beads), conjugated with capture antibodies that are used 


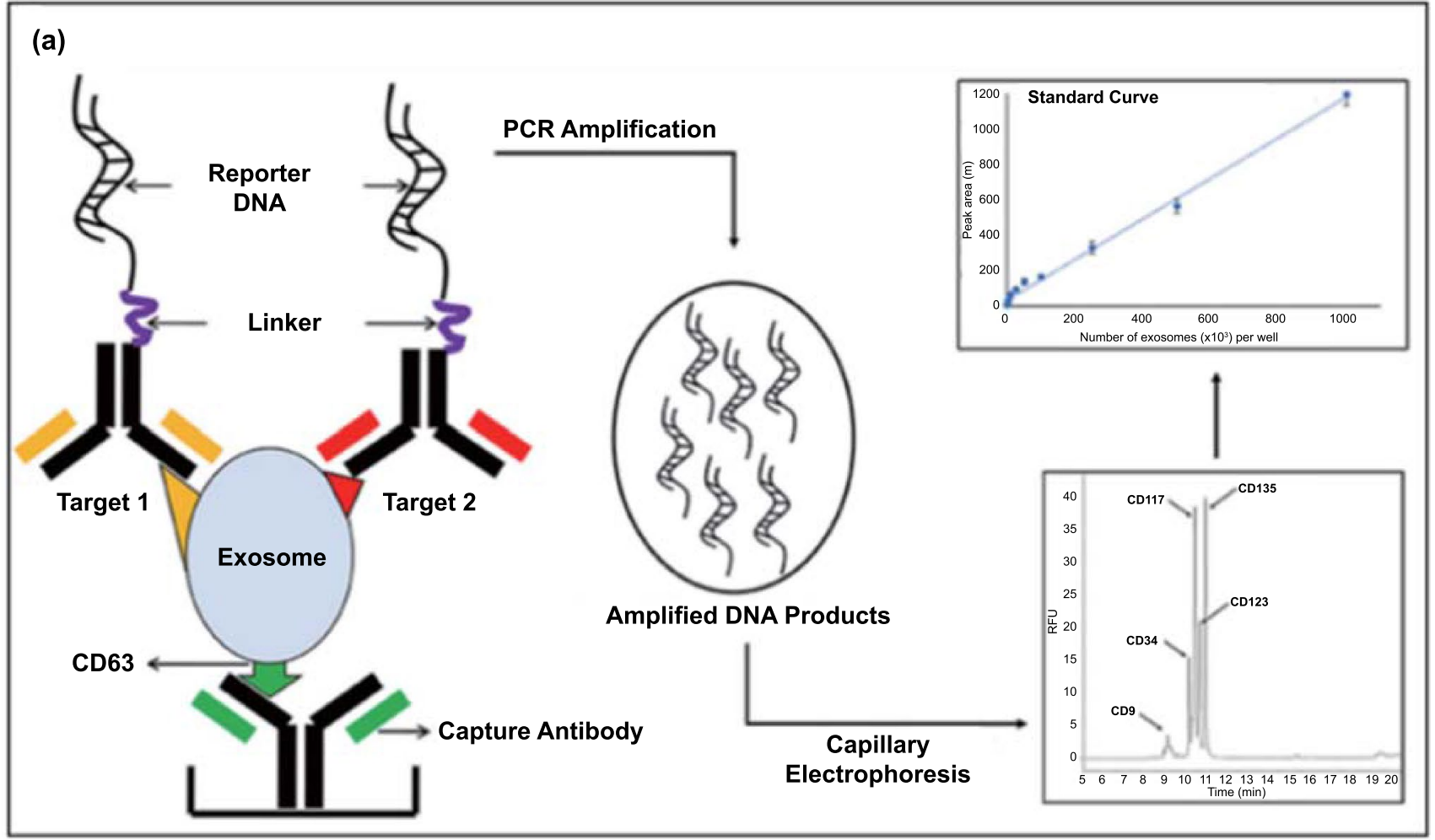

(b)

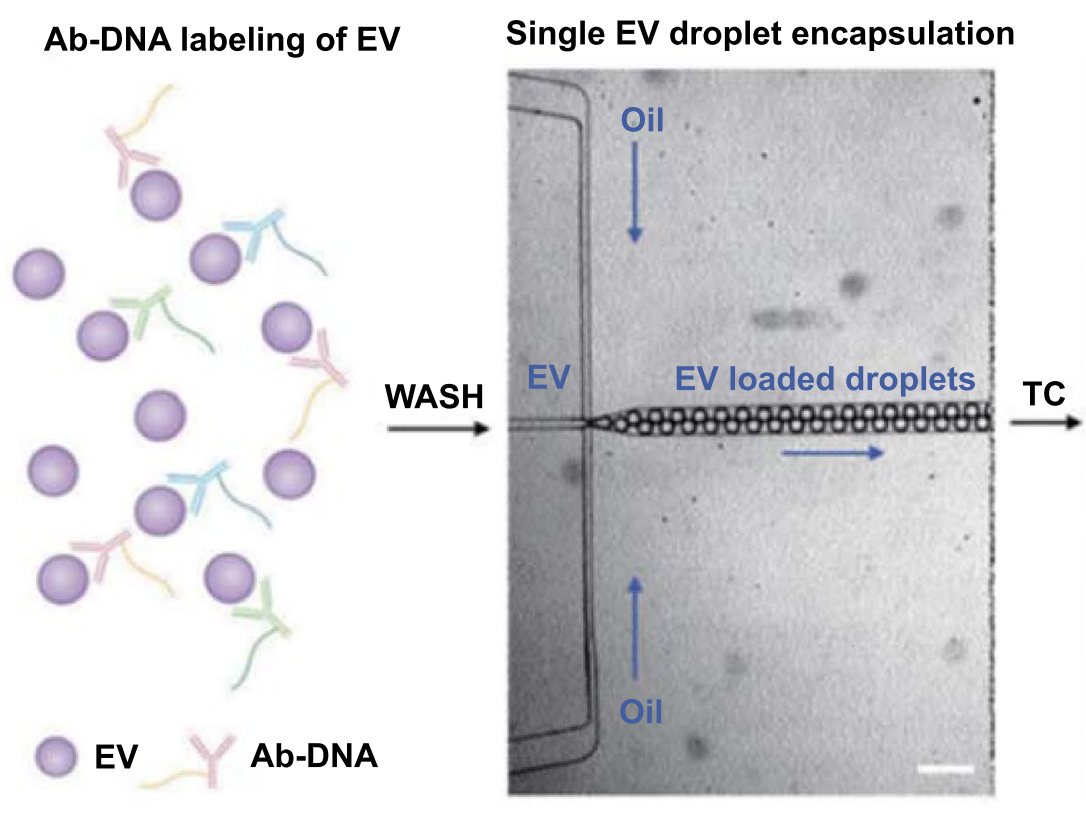

Fig. 9 a Schematic assay format of immuno-PCR assisted multiplex detection of membrane proteins on EVs using capillary electrophoresis. The standard curve of the peak area in an electropherogram vs number of exosomes per well for multiplex immuno-PCR (upper inset). The immuno-PCR peaks for the detection of CD9, CD34, CD117, CD123, and CD135 molecules (bottom inset). Adapted with permission from Ref. [155]. Copyright 2020 American Chemical Society. b The convergence of antibody-DNA labelling and digital PCR for EV multiplexing. Reproduced with permission from Ref. [157]. Copyright 2020 John Wiley and Sons 
Table 2 Summary of the different EV multiplexing strategies

\begin{tabular}{|c|c|c|c|}
\hline $\begin{array}{l}\text { EV mul- } \\
\text { tiplexing } \\
\text { strategies }\end{array}$ & Principle & Advantages & Limitations \\
\hline Chemical & $\begin{array}{l}\text { Use of multiple chemical labels to code } \\
\text { different EV analytes }\end{array}$ & $\begin{array}{l}\text { Detection of specific EV analytes using } \\
\text { commercially available chemical labels } \\
\text { (e.g. optical dyes, redox probes) }\end{array}$ & $\begin{array}{l}\text { Limited choice of chemical labels, over- } \\
\text { lapping spectra or redox peaks }\end{array}$ \\
\hline Physical & $\begin{array}{l}\text { Integration of spatially isolated solid sup- } \\
\text { port }(e . g . \text { array) with multiple receptor } \\
\text { targeting EV analytes }\end{array}$ & $\begin{array}{l}\text { Compatible with high-throughput } \\
\text { measurements in a label-free manner } \\
\text { or specific EV marker detection using } \\
\text { chemical labels. No potential interac- } \\
\text { tions between receptors in liquid phase }\end{array}$ & Usually limited to qualitative assessment \\
\hline Biological & $\begin{array}{l}\text { Use of biomolecules such as DNA to } \\
\text { code EV analytes }\end{array}$ & $\begin{array}{l}\text { Dramatic signal enhancement by nucleic } \\
\text { acid amplification, using relevant meth- } \\
\text { odologies such as PCR }\end{array}$ & $\begin{array}{l}\text { Multiple pre-conjugation steps and } \\
\text { purification of receptor-biomolecule } \\
\text { conjugates are needed }\end{array}$ \\
\hline Nanoparticle & $\begin{array}{l}\text { Nanoparticles with distinct optical or } \\
\text { electrochemical properties used as } \\
\text { labelling tags for signal detection }\end{array}$ & Wide choices of barcoded beads & $\begin{array}{l}\text { Precise tuning of the optical properties of } \\
\text { nanoparticle labels is needed }\end{array}$ \\
\hline
\end{tabular}
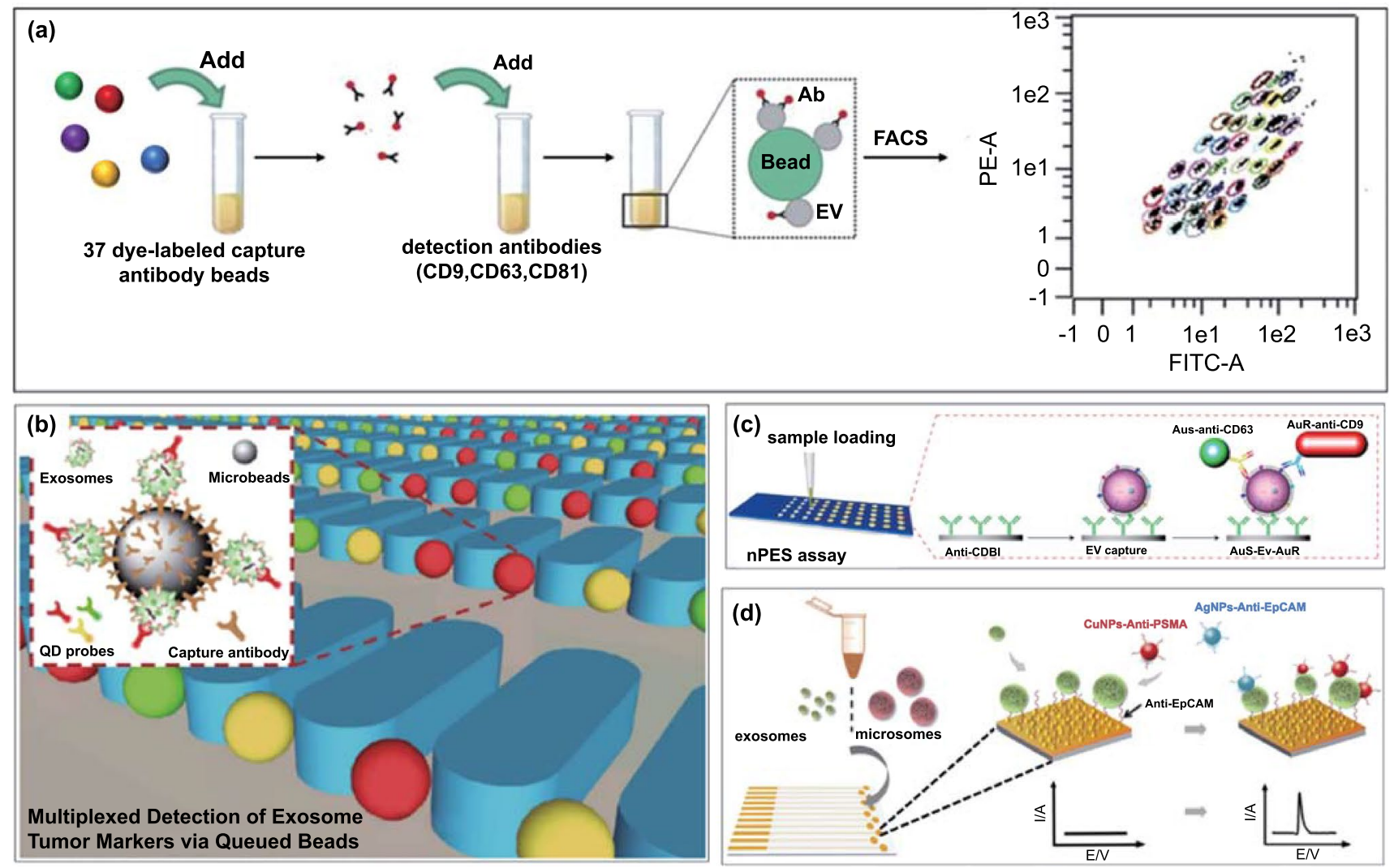

Fig. 10 Nanoparticle coding strategy for EV multiplexing using a fluorophore doped beads. Reproduced with permission from Ref. [165]. Copyright 2020 AAN Publications. b Quantum dots. Reproduced with permission from Ref. [185]. Copyright 2019 Springer. c Plasmonic nanoparticles. Reproduced with permission from Ref. [177]. Copyright 2017 Springer Nature. d Redox active Cu and Ag nanoparticles. Reproduced with permission [179]. Copyright 2014 John Wiley \& Sons 
for multiplexed immunoassays. Shi et al., for example, have employed the Luminex platform to study potential EV biomarkers in Parkinson's disease (PD) [163]. They demonstrated that $\mathrm{EV}$-associated $\alpha$-synuclein is significantly higher in PD patients as compared to controls with an AUC of 0.724 (sensitivity $=76.8 \%$, specificity $=53.5 \%$ ). More recently, Vacchi et al. have used the FCM platform to explore $37 \mathrm{EV}$ surface markers using the plasma-derived EV from PD, healthy controls (HC), multiple system atrophy (MSA), and Atypical Parkinsonism (AP) with tauopathy (AP-Tau) [165]. Although they used a small number of cases, this work demonstrated that the combination of $\mathrm{CD}$ 2, CD62P and CD146 could clearly separate MSA from HC $(\mathrm{AUC}=0.961$, sensitivity $=100 \%$, specificity $=84.2 \%)$.

It is noteworthy that aggregation of EV-bead complexes may complicate assays and lead to biased results. Nonspecific signals, additionally, remain another issue [166]. Encouragingly, new variations of flow cytometry have been developed, for example, the newly developed nano-FCM to facilitate profiling with higher resolution $(\sim 30 \mathrm{~nm})$ and sensitivity $[167,168]$.

\subsection{Quantum Dots}

Quantum dots (QDs) are relatively easy to synthesize with highly tunable optical properties and have accordingly been explored as optical tags for EV profiling. In a recent study, Bai et al., for example, developed a beadbased microarray for EV isolation and multiplexed tumour marker detection. EVs were isolated with anti-CD9 antibody-coated polystyrene microbeads, and tumour markers on the captured EVs were detected with antibodies decorated with quantum dot probes (i.e. $\mathrm{QD}_{525 \mathrm{~nm}}, \mathrm{QD}_{585 \mathrm{~nm}}$, $\mathrm{QD}_{625 \mathrm{~nm}}$ ) as shown in Fig. 10b supporting the detection of the EV proteins CEA, Cyfra21-1, and ProGRP. Using this assay, lung cancer-derived samples were observed to exhibit between 6- to 10-fold higher fluorescence intensity than endothelial cell samples, and different types of lung cancer samples showed distinct marker expression. The antibody-QD coding-based platform offers clear advantages over the conventional organic fluorophores in terms of broader excitation and more sharply defined emission signatures [169-171]. Antibody-conjugated quantum dots (i.e. $\mathrm{QDs}_{605^{-}}$anti-EpCAM and $\mathrm{QDs}_{655^{-}}$anti-EphA2) have also been employed with a lipophilic fluorescent dye $\mathrm{DiO}$ (3,3'-dihexadecyloxacarbocyanine perchlorate) reference stain to provide a quantitative readout. This introduction of normalization enabled the quantification of EV biomarkers in pancreatic cancer with higher performance. The main drawbacks of such immunostaining-based multiplexed analysis are the needs for repeated staining and the generation of signals distinct from nonspecific fluorescence.

\subsection{Plasmonic Nanoparticles}

In addition to SPR, a number of localized SPR (LSPR) platforms have been applied to EVs [108, 172-174]. LSPR results from the confinement of a strong electromagnetic field exhibited by a noble metal in its nanostructure form $[175,176]$ and is highly sensitive to the local refractive index variation adjacent to the metallic surface. For example, Liang et al. have reported a sensitive and specific method to detect pancreatic cancer EV markers by using nanoplasmonenhanced scattering (nPES) sensor (Fig. 10c) [177]. Specifically, anti-EphA2-modified AuNPs and anti-CD9-modified AuNRs were bound to the pre-captured EVs on an antiCD81 coated chip surface to simultaneously assess for the presence of both membrane proteins. When the two nanoparticles were in close proximity $(<10 \mathrm{~nm})$, their scattering spectra shifted and could be analysed by dark field microscopy. This coding can detect $\mathrm{EV}$ proteins down $0.2 \mathrm{ng} \mu \mathrm{L}^{-1}$ from as little as $1 \mu \mathrm{L}$ of plasma. In this work, the authors demonstrate that the nPES assay using EphA2-EVs can distinguish pancreatic cancer from pancreatitis and healthy subjects or used to stage tumour progression and detect early responses to neoadjuvant therapy. The proposed nPES platform offers an attractive means for a rapid, purificationfree and ultrasensitive assessment of EVs. Major challenges here are associated with the tuning of scattering properties (e.g. shape and size). In this regard, photonic materials with tunable structural colours that have not been applied in EV profiling could offer a solution [178].

\subsection{Electroactive Nanoparticles}

Electroactive nanoparticles bearing distinct redox properties have also been utilized as tags for EV multiplexing. For example, Zhou et al. reported a microfabricated microfluidic sensor chip containing eleven individual gold electrodes that enable multiple readouts (Fig. 10d) [179]. 
Instead of using immunomagnetic beads for pre-enrichment of EVs within or outside the fluidic channel, the gold electrodes were directly modified with thiolated anti-EpCAM aptamer to bind EVs. Two metal nanoparticle-based redox labels, anti-EpCAM aptamer-modified silver nanoparticles (AgNPs) and anti-PSMA (prostate-specific membrane antigen) aptamer-modified copper nanoparticles (CuNPs), were applied as labels. Taking advantage of their distinct oxidation potentials (AgNPs, $+350 \mathrm{mV}$ vs. $\mathrm{Ag} / \mathrm{AgCl}$, while the $\mathrm{CuNPs},+600 \mathrm{mV}$ vs. $\mathrm{Ag} / \mathrm{AgCl})$, the direct electrooxidation of both nanoparticles generated distinct electrochemical peaks through which EV markers could be quantified. This platform exhibited a limit of detection (LOD) of $50 \mathrm{EV}$ s/sensor with a low sample input $(25 \mu \mathrm{L})$ and a fast response ( $\leq 10 \mathrm{~s})$ and was used to show that increased levels of PSMA and EpCAM exist in EVs from prostate cancer positive serum samples. This concept has been extended to the analysis of cancer cells by electrochemical oxidation of metal nanoparticle tags, including $\mathrm{Ag}, \mathrm{Cu}$, and $\mathrm{Pd}$ [180]. A similar molecular redox probe based approach has also been explored in electrochemical multiplexing. For example, separately labelled redox probes (Nile blue, ferrocene, and methylene blue with redox peak at $-0.4,-0.2$, and $0.2 \mathrm{~V}$ in single sweep) to graphene oxides tailored with detection antibodies to function as electrochemical signal tags in the sandwich assays were used for multiple cytokine sensing (IL-6, IL-1 $\beta$, and TNF- $\alpha$ ) $[181,182]$. Guo et al. synthesized different ruthenium complexes to achieve an immunosensor for multiplexing cancer biomarkers (CEA, AFP, and beta-human chorionic gonadotropin) [183]. This work has extended the scope of electrochemical multiplexing and is highly adaptable for the detection of EV markers [184]. Like the optical equivalent, the limitation of this approach is the number of spectrally (electrochemically) distinct labels conveniently available.

\section{Conclusions and Outlook}

EV content such as proteins and RNA is emerging as a potentially potent source of biomarkers across diseases including cancer, cardiovascular, and neurological diseases. The development of multiplexing platforms offers a powerful toolbox to enable their clinical application. The existing strategies, i.e. chemical-, physical-, biological-, nanoparticlebased and their convergence, have been systemically reviewed and compared herein. The past decade has witnessed great progress in EV isolation and analysis, with a substantial push towards real clinical translation [186, 187], a push that is much more likely to be realized if robust multiplexed analyses can be developed and commercially scaled. Figure 11 summarises the potential use of multiplexed platforms at the different stages of EV biomarker development: these include highthroughput screening of EV biomarker candidates $(n>10)$, validation of the candidates using multiple platforms or cohorts, and low-throughput devices for the use of validated EV markers in routine clinical practice. It is notable that most methodologies highlighted here have been developed within the last few years and tested mostly in pilot studies involving only a small number of patient samples. To the best of our knowledge, no EV biomarker is yet to enter routine clinical practice. Although the field remains in its infancy, its promise is profound. Below, we discuss the main challenges in clinical translation and the potential solutions to tackle them.

\subsection{Assay Standardization}

Given the diversity of detection techniques discussed in earlier sections (e.g. ECL, electrochemistry, SPR, SERS, fluorescence), assay variation and standardization across laboratories is important and remains problematic. Multiplexed platforms for the analysis of clinical samples may, additionally, suffer from variability in the quality of reagents provided from different suppliers. For example, the affinity constant (i.e. association constant) of antibodies, which may vary depending on the production method, is a key factor that influences assay sensitivity [133]. Thus, high-quality control for biologics (e.g. well-characterized antibodies from the same clone) and batch-to-batch assessment of binding parameters should be available to increase reproducibility. This also requires stringent quality control within each assay, such as the consistent detection of a generic EV marker within each sample. Such surface or internal EV markers often vary between laboratories and a broad consensus could aid the interpretation of disease-associated markers. This is especially relevant for EV biomarker validation by different laboratories using the same or different detection platforms. In addition, the quality of biosamples (e.g. blood, CSF, urine) used to extract EVs may vary between cohorts or centres due to issues at collection (e.g. haemolysis during serum extraction or blood contamination of CSF), transport 


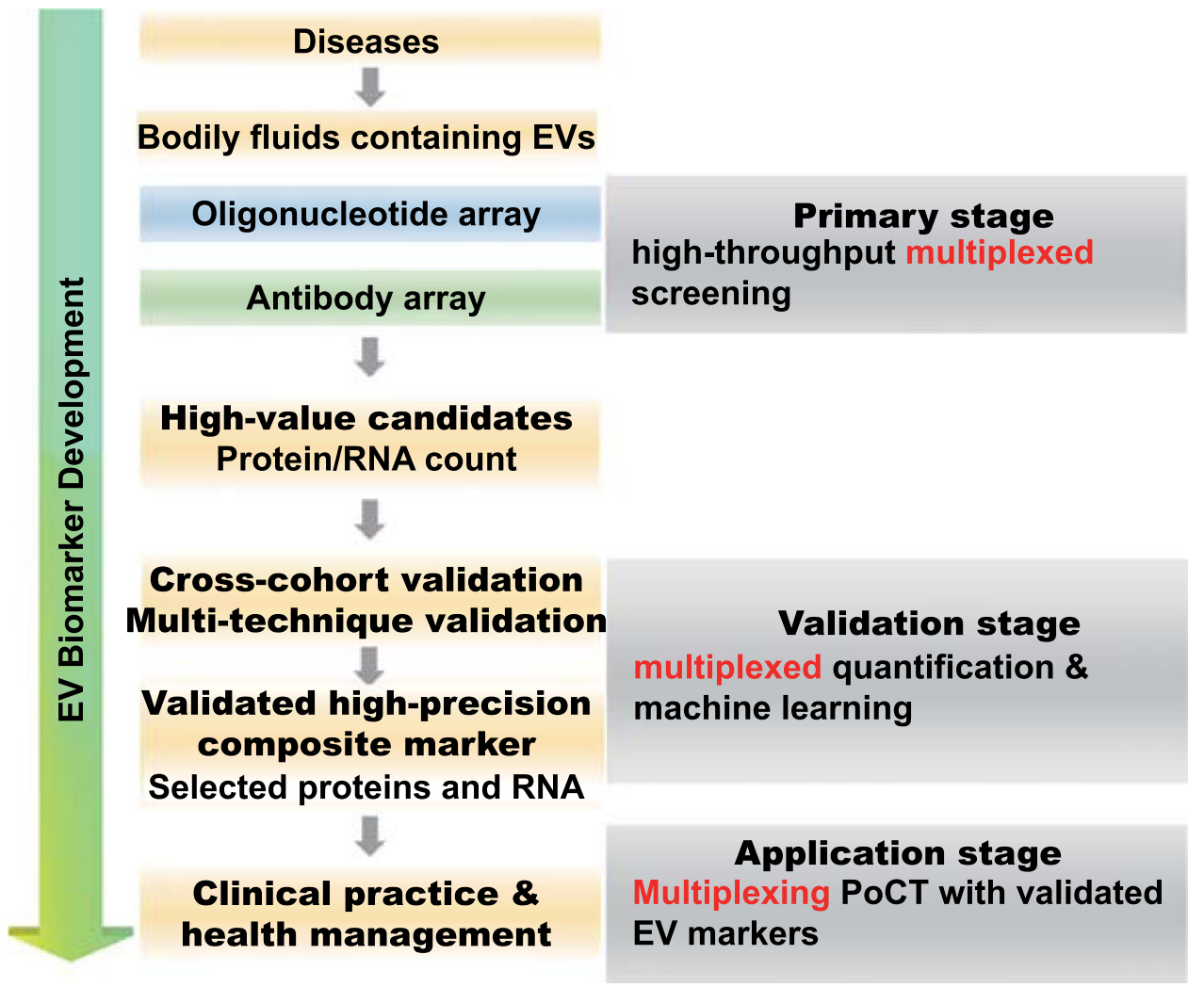

Fig. 11 Flowchart of EV biomarker development powered by multiplexing platform

and storage time-lapse or freeze-thaw cycles. Sample processing and quality control information should be rigorously reported in published studies. To date, most benchtop detection platforms are solely proof-of-concept demonstrations which rely mostly on complicated fabrication steps of the sensor and a series of optimizations performed in a wellequipped centralized facility. Hence, standardized design and fabrication protocols are needed to translate benchtop assays into bedside clinical applications [186].

\subsection{More Adaptive EV Multiplexing Strategies}

\subsubsection{Barcoding System}

Although the concept of barcoding has been used in several EV studies (see Sect. 6.1), the high coding capacity of barcodes in labelling could revolutionise multiplexed assays and high-throughput detection. Barcode technology with facile manipulation of the barcode particle composition to endow spectroscopic, graphical, electronic, and physical codes can be employed for labelling numerous target analytes with distinguishable signal readout $[187,188]$. For clinical application, barcodes serve as reporters to label multiple EV targets, thus offering a promising alternative to traditional labels for EV biomarker identification and discovery [189].

\subsubsection{Chemical Nose System}

Rather than using conventional bio-receptors like antibodies or aptamers, a panel of well-designed chemical ligands, e.g. cationic thiolated-AuNPs and anionic fluorescent polymer-based transducer-based complexations, could be used [190-192]. These chemical ligands contact targets generating optical signals through ligand-target interaction induced displacement, making it a promising means of evaluating EV membrane proteins especially. The latest version of such a chemical ligand system (AIE system) consists of a panel of tetraphenylethene (TPE) derivatives [193, 194] to facilitate EV profiling. 


\subsubsection{CRISPR/Cas System}

The genome editing capacity of the CRISPR/Cas system has been investigated as a novel multiplexing platform [195-199]. Owing to the inherent programmability of Cas proteins [200], collateral activity upon multiple 20-mer barcodes can be extended to report potentially hundreds of orthogonal codes. A proof-of-concept study using CRISPR-Cas12a-mediated barcodes has been applied in the detection of urine biomarkers [197]. Massively multiplexed nucleic acid detection with Cas13-based SHERLOCK detection technology along with microfluidic chips has also been applied to both COVID-19 and other viral infections [201]. Another recent multiplexing platform combining barcoding and dCas 9 has been successfully applied to multiple bacteria DNA detection [202], suggesting that these configurations have high enough flexibility and adaptability to underpin sophisticated EV analysis [200, 203, 204].

\subsection{Advanced Fabrication-Assisted High-throughput and PoC Devices}

High-throughput EV profiling platforms for screening multiple analytes from numerous subjects and the low-throughput PoC devices for the detection of validated EV biomarkers are the important bottlenecks in EV biomarker development (Fig. 11). Their fabrication can, in large part, be readily solved and accelerated by advanced manufacturing [73, 205-207], e.g. photolithography-based micro-/nanofabrication, micro-electro-mechanical systems (MEMS), and 3D printing [208-211] which can integrate different functional modules (isolation, high-throughput detection) into a sample handling microfluidic devices. The most common formats of this kind have enabled the integration of $\mathrm{EV}$ isolation and detection as applied to membrane marker profiling, but rarely for internal EV marker measurements since an additional lysis step is needed [212, 213]. A promising alternative method to realize in situ isolation-lysis-detection could involve the incorporation of an acoustic [214, 215] or an electric field [156] module to realize the goal of "sample-in, result-out" [216, 217] within a single miniaturized device. The on-site acoustic wave lysis might be a good option to avoid the injection of a lysis buffer to the channel. This is potentially easier to operate and has been used for on-chip EV RNA analysis [215]. This "all-in-one" strategy can undoubtedly improve reproducibility by eliminating the manual handling of EV isolation before introducing them into the multiplexing device as summarized in Table 1 . The integration of more sophisticated nonfouling chemistries on device interfaces should reduce false positive/negative results, especially as applied to complex samples $[113,132$, 218]. The investigation of multi-dimensional markers such as RNA and proteins in one device is also of high significance. This can further reduce the handling variations across multiple detection platforms. The emerging integration of powerful algorithms could combine surface signatures and internal cargo profiles, offering more comprehensive profiling of $\mathrm{EV}$ markers in disease.

\subsection{High-Performance Composite Markers and Machine Learning}

It is becoming clear that a combination of biomarker candidates is more likely to generate a precise readout in complex diseases since single markers have failed to achieve this objective in most studies. Composite biomarkers are expected to better reflect disease stratification or progression and provide precision diagnosis in prodromal stages. For example, we have recently demonstrated that the composite measurement of $\alpha$-synuclein and clusterin in serum L1CAM-positive EVs was highly accurate $(\mathrm{AUC}=0.98)$ in differentiating Parkinson's disease from atypical parkinsonism using 735 samples from four independent cohorts. This was superior to the performance of the corresponding individual markers ( AUC 0.82-0.86) [93]. Another report has shown that the detection of EGFR activating and T790M mutations in EV RNA and free circulating DNA offers clinically meaningful sensitivity $(>0.9)$ for lung cancer studies [219]. Advanced data analysis will be important in maximizing the impact of multiplexed measurements across large cohorts. Machine learning has been widely used to maximise the benefits of multi-dimensional markers, e.g. multi-omicsbased biomarkers [220-222]. It offers a powerful means to handle big data especially when dealing with multiplexed profiling of individual samples from hundreds of patients. The application of advanced algorithms capable of studying the logical relationship between EV biomarkers and disease will facilitate the development of high-performance composite marker patterns. 


\subsection{Multiplexing at Single-EV Level}

A more detailed mapping of the surface proteins of EV subpopulations and the development of single-EV level multiplexing platforms will revolutionise the field. This powerful combination will enable an in-depth study of EV heterogeneity and, more importantly, will provide the sensitivity and resolution needed for precise monitoring of the diverse pathological processes that underpin complex diseases. Recent studies have started to investigate multiple membrane proteins or lipid content at single-EV level [51, 223]. It is worth noting that, although single-cell proteomics and sequencing have been realized, the translation of these technologies to single-EV measurements is currently challenging due to trace levels of their cargoes. The improvement of assay sensitivity will undoubtedly support breakthroughs in this area.

The development of multiplexing platforms with diverse strategies has enabled the discovery and quantitation of promising EV biomarkers over the past decade, but their translation into routinely used clinical tests is yet to be fully realized. The latter will require further standardization of current methods and higher resolution potentially at single particle level to capture EV heterogeneity and their precise association with complex diseases.

Acknowledgements Our research is funded by grants from the EPSRC (EP/M006204/1), the Michael J Fox Foundation, the Selfridges Group Foundation and the NIHR Oxford Biomedical Research Centre to G.K.T and J.J.D. The opinion expressed and arguments employed herein do not necessarily reflect the official views of these funding bodies. CJ received support from the John Fell Fund (HMD00470).

Funding Open access funding provided by Shanghai Jiao Tong University.

Open Access This article is licensed under a Creative Commons Attribution 4.0 International License, which permits use, sharing, adaptation, distribution and reproduction in any medium or format, as long as you give appropriate credit to the original author(s) and the source, provide a link to the Creative Commons licence, and indicate if changes were made. The images or other third party material in this article are included in the article's Creative Commons licence, unless indicated otherwise in a credit line to the material. If material is not included in the article's Creative Commons licence and your intended use is not permitted by statutory regulation or exceeds the permitted use, you will need to obtain permission directly from the copyright holder. To view a copy of this licence, visit http://creativecommons.org/licenses/by/4.0/.

\section{References}

1. M. Colombo, G. Raposo, C. Théry, Biogenesis, secretion, and intercellular interactions of exosomes and other extracellular vesicles. Annu. Rev. Cell Dev. Biol. 3, 255-289 (2014). https://doi.org/10.1146/annurev-cellb io-101512-122326

2. Y.P. Loh, Advances in EV isolation technology and function. Extracell. Vesicles Circ. Nucleic Acids 2(1), 1-2 (2021). https://doi.org/10.20517/evena.2021.09

3. Z. Fan, J. Yu, J. Lin, Y. Liu, Y. Liao, Exosome-specific tumor diagnosis via biomedical analysis of exosome-containing microrna biomarkers. Analyst 144(19), 5856-5865 (2019). https://doi.org/10.1039/C9AN00777F

4. E. Ragni, C.P. Orfei, A. Papait, L. Girolamo, Comparison of miRNA cargo in human adipose-tissue Vs amnioticmembrane derived mesenchymal stromal cells extracellular vesicles for osteoarthritis treatment. Extracell. Vesicles Circ. Nucleic Acids 2(3), 202-221 (2021)

5. H. Xiong, Z. Huang, Z. Yang, Q. Lin, B. Yang et al., Recent progress in detection and profiling of cancer cell-derived exosomes. Small 17(35), 2007971 (2021). https://doi.org/ 10.1002/smll.202007971

6. S. Cui, Z. Cheng, W. Qin, L. Jiang, Exosomes as a liquid biopsy for lung cancer. Lung Cancer 116, 46-54 (2018). https://doi.org/10.1016/j.lungcan.2017.12.012

7. T. Hu, J. Wolfram, S. Srivastava, Extracellular vesicles in cancer detection: hopes and hypes. Trends in Cancer 7(2), 122-133 (2021). https://doi.org/10.1016/j.trecan.2020.09.003

8. Z. Fan, K. Xiao, J. Lin, Y. Liao, X. Huang, Functionalized DNA enables programming exosomes/vesicles for tumor imaging and therapy. Small 15(47), 1903761 (2019). https:// doi.org/10.1002/smll.201903761

9. T. Tamura, Y. Yoshioka, S. Sakamoto, T. Ichikawa, T. Ochiya, Extracellular vesicles as a promising biomarker resource in liquid biopsy for cancer. Extracell. Vesicles Circ. Nucleic Acids 2(2), 148-174 (2021). https://doi.org/10.20517/evcna. 2021.06

10. Y. Zhang, N. Ding, S. Xie, Y. Ding, M. Huang et al., Identification of important extracellular vesicle RNA molecules related to sperm motility and prostate cancer. Extracell. Vesicles Circ. Nucleic Acids 2(2), 104-126 (2021). https://doi. org/10.20517/evena.2021.02

11. P.R. Tomlinson, Y. Zheng, R. Fischer, R. Heidasch, C. Gardiner et al., Identification of distinct circulating exosomes in Parkinson's disease. Ann. Clin. Transl. Neurol. 2(4), 353-361 (2015). https://doi.org/10.1002/acn3.175

12. Q. Li, G.K. Tofaris, J.J. Davis, Concentration-normalized electroanalytical assaying of exosomal markers. Anal. Chem. 89(5), 3184-3190 (2017). https://doi.org/10.1021/acs.analc hem.6b05037

13. G.K. Tofaris, A critical assessment of exosomes in the pathogenesis and stratification of Parkinson's disease. J. Parkinsons. Dis. 7(4), 569-576 (2017). https://doi.org/10.3233/ JPD-171176 
14. L. Xiao, S. Hareendran, Y.P. Loh, Function of exosomes in neurological disorders and brain tumors. Extracell. Vesicles Circ. Nucleic Acids 2(1), 55-79 (2021). https://doi.org/10. 20517/evena.2021.04

15. C. Jiang, F. Hopfner, D. Berg, M.T. Hu, A. Pilotto et al., Validation of $\alpha$-synuclein in L1CAM-immunocaptured exosomes as a biomarker for the stratification of parkinsonian syndromes. Mov. Disord. (2021). https://doi.org/10.1002/mds. 28591

16. Y. Bei, S. Das, R.S. Rodosthenous, P. Holvoet, M. Vanhaverbeke et al., Extracellular vesicles in cardiovascular theranostics. Theranostics 7(17), 4168-4182 (2017). https://doi.org/ 10.7150/thno. 21274

17. F. Jansen, G. Nickenig, N. Werner, Extracellular vesicles in cardiovascular disease. Circul. Res. 120(10), 1649-1657 (2017). https://doi.org/10.1161/CIRCRESAHA.117.310752

18. H. Shao, H. Im, C.M. Castro, X. Breakefield, R. Weissleder et al., New technologies for analysis of extracellular vesicles. Chem. Rev. 118(4), 1917-1950 (2018). https://doi.org/ 10.1021/acs.chemrev.7b00534

19. H. Xu, B.C. Ye, Advances in biosensing technologies for analysis of cancer-derived exosomes. TrAC Trends Anal. Chem. 123, 115773 (2020). https://doi.org/10.1016/j.trac. 2019.115773

20. S. Zhou, Y. Yang, Y. Wu, S. Liu, Review: multiplexed profiling of biomarkers in extracellular vesicles for cancer diagnosis and therapy monitoring. Anal. Chim. Acta 1175, 338633 (2021). https://doi.org/10.1016/j.aca.2021.338633

21. A. Nakamura, N. Kaneko, V.L. Villemagne, T. Kato, J. Doecke et al., High performance plasma amyloid-B biomarkers for Alzheimer's disease. Nature 554(7691), 249254 (2018). https://doi.org/10.1038/nature25456

22. D. Jin, X.X. Peng, Y. Qin, P. Wu, H. Lu et al., Multivalence-actuated DNA nanomachines enable bicolor exosomal phenotyping and PD-L1-guided therapy monitoring. Anal. Chem. 92(14), 9877-9886 (2020). https://doi.org/10. 1021/acs.analchem.0c01387

23. Q. Chen, T. Sun, C. Jiang, Recent advancements in nanomedicine for 'cold' tumor immunotherapy. NanoMicro Lett. 13, 92 (2021). https://doi.org/10.1007/ s40820-021-00622-6

24. M. Zhang, K. Jin, L. Gao, Z. Zhang, F. Li et al., Methods and technologies for exosome isolation and characterization. Small Methods 2(9), 1800021 (2018). https://doi.org/10. 1002/smtd.201800021

25. P. Ziaei, C.E. Berkman, M.G. Norton, Isolation and detection of tumor-derived extracellular vesicles. ACS Appl. Nano Mater. 1(5), 2004-2020 (2018). https://doi.org/10.1021/ acsanm.8b00267

26. Z. Zhao, H. Wijerathne, A.K. Godwin, S.A. Soper, Isolation and analysis methods of extracellular vesicles (EVs). Extracell. Vesicles Circ. Nucleic Acids 2(1), 80-103 (2021). https://doi.org/10.20517/evcna.2021.07

27. A. Abramowicz, P. Widlak, M. Pietrowska, Proteomic analysis of exosomal cargo: the challenge of high purity vesicle isolation. Mol. Biosyst. 12(5), 1407-1419 (2016). https://doi. org/10.1039/C6MB00082G

28. D.S. Choi, D.K. Kim, Y.K. Kim, Y.S. Gho, Proteomics of extracellular vesicles: exosomes and ectosomes. Mass Spectrom. Rev. 34(4), 474-490 (2015). https://doi.org/10.1002/ mas. 21420

29. S. Wu, Y. Li, W. Ding, L. Xu, Y. Ma et al., Recent advances of persistent luminescence nanoparticles in bioapplications. Nano-Micro Lett. 12, 70 (2020). https://doi.org/10.1007/ s40820-020-0404-8

30. C. Zong, M. Xu, L.J. Xu, T. Wei, X. Ma et al., Surfaceenhanced Raman spectroscopy for bioanalysis: reliability and challenges. Chem. Rev. 118(10), 4946-4980 (2018). https:// doi.org/10.1021/acs.chemrev.7b00668

31. J. Plou, I. García, M. Charconnet, I. Astobiza, C. GarcíaAstrain et al., Multiplex SERS detection of metabolic alterations in tumor extracellular media. Adv. Funct. Mater. 30(17), 1910335 (2020). https://doi.org/10.1002/adfm.201910335

32. Y. Zheng, C. Jiang, S.H. Ng, Y. Lu, F. Han et al., Unclonable plasmonic security labels achieved by shadow-mask-lithography-assisted self-assembly. Adv. Mater. 28(12), 2330-2336 (2016). https://doi.org/10.1002/adma.201505022

33. Z. Ji, C. Zhang, Y. Ye, J. Ji, H. Dong et al., Magnetically enhanced liquid SERS for ultrasensitive analysis of bacterial and SARS-Cov-2 biomarkers. Front. Bioeng. Biotechnol. 9(787), 735711 (2021). https://doi.org/10.3389/fbioe. 2021.735711

34. Y. Wang, S. Zeng, A. Crunteanu, Z. Xie, G. Humbert et al., Targeted sub-attomole cancer biomarker detection based on phase singularity 2D nanomaterial-enhanced plasmonic biosensor. Nano-Micro Lett. 13, 96 (2021). https://doi.org/ 10.1007/s40820-021-00613-7

35. H. Shin, H. Jeong, J. Park, S. Hong, Y. Choi, Correlation between cancerous exosomes and protein markers based on surface-enhanced Raman spectroscopy (SERS) and principal component analysis (PCA). ACS Sens. 3(12), 26372643 (2018). https://doi.org/10.1021/acssensors.8b01047

36. W. Zhang, L. Jiang, R.J. Diefenbach, D.H. Campbell, B.J. Walsh et al., Enabling sensitive phenotypic profiling of cancer-derived small extracellular vesicles using surfaceenhanced Raman spectroscopy nanotags. ACS Sens. 5(3), 764-771 (2020). https://doi.org/10.1021/acssensors.9b023 77

37. Z. Wang, S. Zong, Y. Wang, N. Li, L. Li et al., Screening and multiple detection of cancerous exosomes using a SERSbased method. Nanoscale 10, 9053-9062 (2018). https://doi. org/10.1039/C7NR09162A

38. C.F. Ning, L. Wang, Y.F. Tian, B.C. Yin, B.C. Ye, Multiple and sensitive SERS detection of cancer-related exosomes based on gold-silver bimetallic nanotrepangs. Analyst 145(7), 2795-2804 (2020). https://doi.org/10.1039/C9AN0 $2180 \mathrm{~A}$

39. J. Wang, A. Wuethrich, A.A.I. Sina, R.E. Lane, L.L. Lin et al., Tracking extracellular vesicle phenotypic changes enables treatment monitoring in melanoma. Sci. Adv. 6(9), eaax3223 (2020). https://doi.org/10.1126/sciadv.aax3223 
40. G. Huang, G. Lin, Y. Zhu, W. Duan, D. Jin, Emerging technologies for profiling extracellular vesicle heterogeneity. Lab Chip 20(14), 2423-2437 (2020). https://doi.org/10.1039/ D0LC00431F

41. D. Cialla, A. März, R. Böhme, F. Theil, K. Weber et al., Surface-enhanced Raman spectroscopy (SERS): progress and trends. Anal. Bioanal. Chem. 403(1), 27-54 (2012). https:// doi.org/10.1007/s00216-011-5631-x

42. D. Cialla-May, X.S. Zheng, K. Weber, J. Popp, Recent progress in surface-enhanced Raman spectroscopy for biological and biomedical applications: from cells to clinics. Chem. Soc. Rev. 46(13), 3945-3961 (2017). https://doi.org/10.1039/ C7CS00172J

43. Z. Zhao, Y. Yang, Y. Zeng, M. He, A microfluidic Exosearch chip for multiplexed exosome detection towards blood-based ovarian cancer diagnosis. Lab Chip 16(3), 489-496 (2016). https://doi.org/10.1039/c5lc01117e

44. M. Deng, C. Jiang, L. Jia, N-Methylimidazolium modified magnetic particles as adsorbents for solid phase extraction of genomic deoxyribonucleic acid from genetically modified soybeans. Anal. Chim. Acta 771, 31-36 (2013). https://doi. org/10.1016/j.aca.2013.02.005

45. Y. Guan, C. Jiang, C. Hu, L. Jia, Preparation of multi-walled carbon nanotubes functionalized magnetic particles by sol-gel technology and its application in extraction of estrogens. Talanta 83(2), 337-343 (2010). https://doi.org/10.1016/j.talanta. 2010.09.023

46. S. Lin, Z. Yu, D. Chen, Z. Wang, J. Miao et al., Progress in microfluidics-based exosome separation and detection technologies for diagnostic applications. Small 16(9), 1903916 (2020). https://doi.org/10.1002/smll.201903916

47. S. Xu, C. Jiang, Y. Lin, L. Jia, Magnetic nanoparticles modified with polydimethylsiloxane and multi-walled carbon nanotubes for solid-phase extraction of fluoroquinolones. Microchim. Acta 179(3), 257-264 (2012). https://doi.org/ 10.1007/s00604-012-0894-2

48. S. Fang, H. Tian, X. Li, D. Jin, X. Li et al., Clinical application of a microfluidic chip for immunocapture and quantification of circulating exosomes to assist breast cancer diagnosis and molecular classification. PLoS ONE 12(4), e0175050 (2017). https://doi.org/10.1371/journal.pone.0175050

49. M. He, J. Crow, M. Roth, Y. Zeng, A.K. Godwin, Integrated immunoisolation and protein analysis of circulating exosomes using microfluidic technology. Lab Chip 14(19), 3773-3780 (2014). https://doi.org/10.1039/C4LC00662C

50. S. Wang, A. Khan, R. Huang, S. Ye, K. Di et al., Recent advances in single extracellular vesicle detection methods. Biosens. Bioelectron. 154, 112056 (2020). https://doi.org/10. 1016/j.bios.2020.112056

51. K. Lee, K. Fraser, B. Ghaddar, K. Yang, E. Kim et al., Multiplexed profiling of single extracellular vesicles. ACS Nano 12(1), 494-503 (2018). https://doi.org/10.1021/acsnano. $7 \mathrm{~b} 07060$

52. S. Cavallaro, F. Pevere, F. Stridfeldt, A. Görgens, C. Paba et al., Multiparametric profiling of single nanoscale extracellular vesicles by combined atomic force and fluorescence microscopy: correlation and heterogeneity in their molecular and biophysical features. Small 17(14), 2008155 (2021). https://doi.org/10.1002/smll.202008155

53. H. Wang, H. Chen, Z. Huang, T. Li, A. Deng et al., DAase I enzyme-aided fluorescence signal amplification based on graphene oxide-DNA aptamer interactions for colorectal cancer exosome detection. Talanta 184, 219-226 (2018). https://doi. org/10.1016/j.talanta.2018.02.083

54. B. Li, C. Liu, W. Pan, J. Shen, J. Guo et al., Facile fluorescent aptasensor using aggregation-induced emission luminogens for exosomal proteins profiling towards liquid biopsy. Biosens. Bioelectron. 168, 112520 (2020). https://doi.org/10. 1016/j.bios.2020.112520

55. Y. Xia, M. Liu, L. Wang, A. Yan, W. He et al., A visible and colorimetric aptasensor based on DNA-capped single-walled carbon nanotubes for detection of exosomes. Biosens. Bioelectron. 92, 8-15 (2017). https://doi.org/10.1016/j.bios.2017. 01.063

56. D. Jin, F. Yang, Y. Zhang, L. Liu, Y. Zhou et al., ExoAPP: exosome-oriented, aptamer nanoprobe-enabled surface proteins profiling and detection. Anal. Chem. 90(24), 1440214411 (2018). https://doi.org/10.1021/acs.analchem.8b03959

57. C. Liu, J. Zhao, F. Tian, L. Cai, W. Zhang et al., Low-cost thermophoretic profiling of extracellular-vesicle surface proteins for the early detection and classification of cancers. Nat. Biomed. Eng. 3(3), 183-193 (2019). https://doi. org/10.1038/s41551-018-0343-6

58. F. Tian, Z. Han, J. Deng, C. Liu, J. Sun, Thermomicrofluidics for biosensing applications. VIEW (2021). https://doi. org/10.1002/VIW.20200148

59. B. Lin, T. Tian, Y. Lu, D. Liu, M. Huang et al., Tracing tumor-derived exosomal PD-L1 by dual-aptamer activated proximity-induced droplet digital PCR. Angew. Chem. Int. Ed. 60(14), 7582-7586 (2021). https://doi.org/10.1002/ anie. 202015628

60. D. Wu, J. Yan, X. Shen, Y. Sun, M. Thulin et al., Profiling surface proteins on individual exosomes using a proximity barcoding assay. Nat. Comm. 10(1), 3854 (2019). https:// doi.org/10.1038/s41467-019-11486-1

61. L. Löf, T. Ebai, L. Dubois, L. Wik, K.G. Ronquist et al., Detecting individual extracellular vesicles using a multicolor in situ proximity ligation assay with flow cytometric readout. Sci. Rep. 6, 34358 (2016). https://doi.org/10.1038/ srep34358

62. J. Zhang, J. Shi, H. Zhang, Y. Zhu, W. Liu et al., Localized fluorescent imaging of multiple proteins on individual extracellular vesicles using rolling circle amplification for cancer diagnosis. J. Extracell. Vesicles 10(1), e12025 (2020). https:// doi.org/10.1002/jev2.12025

63. X. Wu, H. Zhao, A. Natalia, C.Z.J. Lim, N.R.Y. Ho et al., Exosome-templated nanoplasmonics for multiparametric molecular profiling. Sci. Adv. 6(19), 2556 (2020). https:// doi.org/10.1126/sciadv.aba2556

64. E.C. Yeh, C.C. Fu, L. Hu, R. Thakur, J. Feng et al., Self-powered integrated microfluidic point-of-care low-cost enabling 
(SIMPLE) chip. Sci. Adv. 3(3), e1501645 (2017). https://doi. org/10.1126/sciadv.1501645

65. V. Yelleswarapu, J.R. Buser, M. Haber, J. Baron, E. Inapuri et al., Mobile platform for rapid sub-picogram-per-milliliter, multiplexed, digital droplet detection of proteins. PNAS 116(10), 4489-4495 (2019). https://doi.org/10.1073/pnas. 1814110116

66. B. Shu, L. Lin, B. Wu, E. Huang, Y. Wang et al., A pocketsized device automates multiplexed point-of-care RNA testing for rapid screening of infectious pathogens. Biosens. Bioelectron. 181, 113145 (2021). https://doi.org/10.1016/j. bios.2021.113145

67. T. Tian, B. Shu, Y. Jiang, M. Ye, L. Liu et al., An ultralocalized cas13a assay enables universal and nucleic acid amplification-free single-molecule RNA diagnostics. ACS Nano 15(1), 1167-1178 (2021). https://doi.org/10.1021/acsnano. 0c08165

68. C.Z.J. Lim, Y. Zhang, Y. Chen, H. Zhao, M.C. Stephenson et al., Subtyping of circulating exosome-bound amyloid $\beta$ reflects brain plaque deposition. Nat. Comm. 10(1), 1144 (2019). https://doi.org/10.1038/s41467-019-09030-2

69. Y. Li, J. Deng, Z. Han, C. Liu, F. Tian et al., Molecular identification of tumor-derived extracellular vesicles using thermophoresis-mediated DNA computation. J. Am. Chem. Soc. 143(3), 1290-1295 (2021). https://doi.org/10.1021/jacs. 0c12016

70. J. Zhao, C. Liu, Y. Li, Y. Ma, J. Deng et al., Thermophoretic detection of exosomal microRNAs by nanoflares. J. Am. Chem. Soc. 142(11), 4996-5001 (2020). https://doi.org/10. 1021/jacs.9b13960

71. H. Schwarzenbach, N. Nishida, G.A. Calin, K. Pantel, Clinical relevance of circulating cell-free microRNAs in cancer. Nat. Rev. Clin. Oncol. 11(3), 145-156 (2014). https://doi.org/ 10.1038/nrclinonc. 2014.5

72. J.R. Chevillet, Q. Kang, I.K. Ruf, H.A. Briggs, L.N. Vojtech et al., Quantitative and stoichiometric analysis of the microRNA content of exosomes. PNAS 111(41), 14888-14893 (2014). https://doi.org/10.1073/pnas.1408301111

73. G. Liu, C. Jiang, X. Lin, Y. Yang, Point-of-care detection of cytokines in cytokine storm management and beyond: significance and challenges. VIEW 2(4), 20210003 (2021). https:// doi.org/10.1002/VIW.20210003

74. J.H. Lee, J.A. Kim, S. Jeong, W.J. Rhee, Simultaneous and multiplexed detection of exosome micrornas using molecular beacons. Biosens. Bioelectron. 86, 202-210 (2016). https:// doi.org/10.1016/j.bios.2016.06.058

75. J. Lee, M.H. Kwon, J.A. Kim, W.J. Rhee, Detection of exosome miRNAs using molecular beacons for diagnosing prostate cancer. Artif. Cells Nanomed. Biotechnol. 46, S52-S63 (2018). https://doi.org/10.1080/21691401.2018.1489263

76. H.C. Yang, W.J. Rhee, Single step in situ detection of surface protein and microRNA in clustered extracellular vesicles using flow cytometry. J. Clin. Med. 10(2), 319 (2021). https:// doi.org/10.3390/jcm10020319

77. S. Zhou, T. Hu, G. Han, Y. Wu, X. Hua et al., Accurate cancer diagnosis and stage monitoring enabled by comprehensive profiling of different types of exosomal biomarkers: surface proteins and miRNAs. Small 16(48), 2004492 (2020). https:// doi.org/10.1002/smll.202004492

78. C. Liu, E. Kannisto, G. Yu, Y. Yang, M.E. Reid et al., Noninvasive detection of exosomal microRNAs via tethered cationic lipoplex nanoparticles (tCLN) biochip for lung cancer early detection. Front. Genet. 11(258), 258 (2020). https:// doi.org/10.3389/fgene.2020.00258

79. Y. Yang, E. Kannisto, G. Yu, M.E. Reid, S.K. Patnaik et al., An immuno-biochip selectively captures tumor-derived exosomes and detects exosomal RNAs for cancer diagnosis. ACS Appl. Mater. Interfaces 10(50), 43375-43386 (2018). https://doi.org/10.1021/acsami.8b13971

80. J. Zhou, Z. Wu, J. Hu, D. Yang, X. Chen et al., High-throughput single-EV liquid biopsy: rapid, simultaneous, and multiplexed detection of nucleic acids, proteins, and their combinations. Sci. Adv. 6(47), eabc1204 (2020). https://doi.org/ 10.1126/sciadv.abc1204

81. Y. Wu, K.J. Kwak, K. Agarwal, A. Marras, C. Wang et al., Detection of extracellular RNAs in cancer and viral infection via tethered cationic lipoplex nanoparticles containing molecular beacons. Anal. Chem. 85(23), 11265-11274 (2013). https://doi.org/10.1021/ac401983w

82. X. Wang, K.J. Kwak, Z. Yang, A. Zhang, X. Zhang et al., Extracellular mRNA detected by molecular beacons in tethered lipoplex nanoparticles for diagnosis of human hepatocellular carcinoma. PLoS ONE 13(6), e0198552 (2018). https://doi.org/10.1371/journal.pone.0198552

83. P. Ghassemi, B. Wang, J. Wang, Q. Wang, Y. Chen et al., Evaluation of mobile phone performance for near-infrared fluorescence imaging. IEEE Trans. Biomed. Eng. 64(7), 1650-1653 (2017). https://doi.org/10.1109/TBME.2016. 2601014

84. R. Vaidyanathan, M. Naghibosadat, S. Rauf, D. Korbie, L.G. Carrascosa et al., Detecting exosomes specifically: a multiplexed device based on alternating current electrohydrodynamic induced nanoshearing. Anal. Chem. 86(22), 11125-11132 (2014). https://doi.org/10.1021/ac502082b

85. C. Liu, J. Zhao, F. Tian, J. Chang, W. Zhang et al., $\lambda$-DNAand aptamer-mediated sorting and analysis of extracellular vesicles. J. Am. Chem. Soc. 141(9), 3817-3821 (2019). https://doi.org/10.1021/jacs.9b00007

86. H. Im, H. Shao, Y.I. Park, V.M. Peterson, C.M. Castro et al., Label-free detection and molecular profiling of exosomes with a nano-plasmonic sensor. Nat. Biotechnol. 32(5), 490-495 (2014). https://doi.org/10.1038/nbt.2886

87. W. Liu, J. Li, Y. Wu, S. Xing, Y. Lai et al., Target-induced proximity ligation triggers recombinase polymerase amplification and transcription-mediated amplification to detect tumor-derived exosomes in nasopharyngeal carcinoma with high sensitivity. Biosens. Bioelectron. 102, 204-210 (2018). https://doi.org/10.1016/j.bios.2017.11.033

88. Y. Jiang, M. Shi, Y. Liu, S. Wan, C. Cui et al., Aptamer/ AuNP biosensor for colorimetric profiling of exosomal proteins. Angew. Chem. Int. Ed. 56(39), 11916-11920 (2017). https://doi.org/10.1002/anie.201703807 
89. Y. Lyu, D. Cui, J. Huang, W. Fan, Y. Miao et al., Nearinfrared afterglow semiconducting nano-polycomplexes for the multiplex differentiation of cancer exosomes. Angew. Chem. Int. Ed. 58(15), 4983-4987 (2019). https://doi.org/ 10.1002/ange.201900092

90. Y.J. Chiu, W. Cai, Y.R.V. Shih, I. Lian, Y.H. Lo, A singlecell assay for time lapse studies of exosome secretion and cell behaviors. Small 12(27), 3658-3666 (2016). https:// doi.org/10.1002/sml1.201600725

91. K. Mori, M. Hirase, T. Morishige, E. Takano, H. Sunayama et al., A pretreatment-free, polymer-based platform prepared by molecular imprinting and post-imprinting modifications for sensing intact exosomes. Angew. Chem. Int. Ed. 58(6), 1612-1615 (2019). https://doi.org/10.1002/anie. 201811142

92. S. Jeong, J. Park, D. Pathania, C.M. Castro, R. Weissleder et al., Integrated magneto-electrochemical sensor for exosome analysis. ACS Nano 10(2), 1802-1809 (2016). https:// doi.org/10.1021/acsnano.5b07584

93. C. Jiang, F. Hopfner, A. Katsikoudi, R. Hein, C. Catli et al., Serum neuronal exosomes predict and differentiate Parkinson's disease from atypical parkinsonism. J. Neurol. Neurosurg. Psychiatry 91, 720-729 (2020). https://doi.org/10.1136/ jnnp-2019-322588

94. J. Park, H. Im, S. Hong, C.M. Castro, R. Weissleder et al., Analyses of intravesicular exosomal proteins using a nanoplasmonic system. ACS Photonics 5(2), 487-494 (2018). https://doi.org/10.1021/acsphotonics.7b00992

95. E.A. Kwizera, R. O'Connor, V. Vinduska, M. Williams, E.R. Butch et al., Molecular detection and analysis of exosomes using surface-enhanced Raman scattering gold nanorods and a miniaturized device. Theranostics 8(10), 2722 (2018). https://doi.org/10.7150/thno.21358

96. L. Zhu, K. Wang, J. Cui, H. Liu, X. Bu et al., Label-free quantitative detection of tumor-derived exosomes through surface plasmon resonance imaging. Anal. Chem. 86(17), 8857-8864 (2014). https://doi.org/10.1021/ac5023056

97. M. Rodrigues, N. Richards, B. Ning, C.J. Lyon, T.Y. Hu, Rapid lipid-based approach for normalization of quantumdot-detected biomarker expression on extracellular vesicles in complex biological samples. Nano Lett. 19(11), 7623-7631 (2019). https://doi.org/10.1021/acs.nanolett.9b02232

98. T. Takeuchi, K. Mori, H. Sunayama, E. Takano, Y. Kitayama et al., Antibody-conjugated signaling nanocavities fabricated by dynamic molding for detecting cancers using small extracellular vesicle markers from tears. J. Am. Chem. Soc. 142(14), 6617-6624 (2020). https://doi.org/10.1021/jacs. $9 b 13874$

99. P. Zhang, X. Zhou, Y. Zeng, Multiplexed immunophenotyping of circulating exosomes on nano-engineered exoprofile chip towards early diagnosis of cancer. Chem. Sci. 10(21), 5495-5504 (2019). https://doi.org/10.1039/C9SC00961B

100. S. Zhou, T. Hu, F. Zhang, D. Tang, D. Li et al., Integrated microfluidic device for accurate extracellular vesicle quantification and protein markers analysis directly from human whole blood. Anal. Chem. 92(1), 1574-1581 (2020). https:// doi.org/10.1021/acs.analchem.9b04852

101. Y. An, R. Li, F. Zhang, P. He, Magneto-mediated electrochemical sensor for simultaneous analysis of breast cancer exosomal proteins. Anal. Chem. 92(7), 5404-5410 (2020). https://doi.org/10.1021/acs.analchem.0c00106

102. K.S. Yang, H. Im, S. Hong, I. Pergolini, A.F. Castillo et al., Multiparametric plasma EV profiling facilitates diagnosis of pancreatic malignancy. Sci. Transl. Med. 9(391), 13226 (2017). https://doi.org/10.1126/scitranslmed.aal3226

103. H.L. Cheng, C.Y. Fu, W.C. Kuo, Y.W. Chen, Y.S. Chen et al., Detecting mirna biomarkers from extracellular vesicles for cardiovascular disease with a microfluidic system. Lab Chip 18(19), 2917-2925 (2018). https://doi.org/10.1039/C8LC0 0386F

104. H. Wang, D. He, K. Wan, X. Sheng, H. Cheng et al., In situ multiplex detection of serum exosomal micrornas using allin-one biosensor for breast cancer diagnosis. Analyst 145, 3289-3296 (2020). https://doi.org/10.1039/D0AN00393J

105. M. Jang, G. Choi, Y.Y. Choi, J.E. Lee, J.H. Jung et al., Extracellular vesicle (EV)-polyphenol nanoaggregates for microrna-based cancer diagnosis. NPG Asia Mater. 11(1), 79 (2019). https://doi.org/10.1038/s41427-019-0184-0

106. Z. Wang, X. Sun, A. Natalia, C.S.L. Tang, C.B.T. Ang et al., Dual-selective magnetic analysis of extracellular vesicle glycans. Matter 2(1), 150-166 (2020). https://doi.org/10.1016/j. matt.2019.10.018

107. D.L.M. Rupert, C. Lässer, M. Eldh, S. Block, V.P. Zhdanov et al., Determination of exosome concentration in solution using surface plasmon resonance spectroscopy. Anal. Chem. 86(12), 5929-5936 (2014). https://doi.org/10.1021/ac500 931f

108. A. Thakur, G. Qiu, S.P. Ng, J. Guan, J. Yue et al., Direct detection of two different tumor-derived extracellular vesicles by SAM-AuNIs LSPR biosensor. Biosens. Bioelectron. 94, 400-407 (2017). https://doi.org/10.1016/j.bios.2017.03.036

109. E.L. Gool, I. Stojanovic, R.B.M. Schasfoort, A. Sturk, T.G. Leeuwen et al., Surface plasmon resonance is an analytically sensitive method for antigen profiling of extracellular vesicles. Clin. Chem. 63(10), 1633-1641 (2017). https://doi.org/ 10.1373/clinchem.2016.271049

110. M.A. Khan, Y. Zhu, Y. Yao, P. Zhang, A. Agrawal et al., Impact of metal crystallinity-related morphologies on the sensing performance of plasmonic nanohole arrays. Nanoscale 12(14), 7577-7585 (2020). https://doi.org/10. 1039/D0NR00619J

111. C. Liu, Y. Yang, Y. Wu, Recent advances in exosomal protein detection via liquid biopsy biosensors for cancer screening, diagnosis, and prognosis. AAPS J. 20(2), 41 (2018). https:// doi.org/10.1208/s12248-018-0201-1

112. S. Taufik, A. Barfidokht, M.T. Alam, C. Jiang, S.G. Parker et al., An antifouling electrode based on electrode-organic layer-nanoparticle constructs: electrodeposited organic layers versus self-assembled monolayers. J. Electroanal. Chem. 779, 229-235 (2016). https://doi.org/10.1016/j.jelechem.2016.01. 031 
113. C. Jiang, G. Wang, R. Hein, N. Liu, X. Luo et al., Antifouling strategies for selective in vitro and in vivo sensing. Chem. Rev. 120(8), 3852-3889 (2020). https://doi.org/10.1021/acs. chemrev.9b00739

114. W. Wu, X. Yu, J. Wu, T. Wu, Y. Fan et al., Surface plasmon resonance imaging-based biosensor for multiplex and ultrasensitive detection of NSCLC-associated exosomal miRNAs using DNA programmed heterostructure of Au-on-Ag. Biosens. Bioelectron. 175, 112835 (2021). https://doi.org/10. 1016/j.bios.2020.112835

115. J.A. Huang, M.Z. Mousavi, Y. Zhao, A. Hubarevich, F. Omeis et al., SERS discrimination of single DNA bases in single oligonucleotides by electro-plasmonic trapping. Nat. Commun. 10(1), 5321 (2019). https://doi.org/10.1038/ s41467-019-13242-x

116. J.A. Huang, M.Z. Mousavi, G. Giovannini, Y. Zhao, A. Hubarevich et al., Multiplexed discrimination of single amino acid residues in polypeptides in a single SERS hot spot. Angew. Chem. Int. Ed. 59(28), 11423-11431 (2020). https://doi.org/10.1002/anie.202000489

117. S. Pan, Y. Zhang, A. Natalia, C.Z.J. Lim, N.R.Y. Ho et al., Extracellular vesicle drug occupancy enables realtime monitoring of targeted cancer therapy. Nat. Nanotechnol. 16(6), 734-742 (2021). https://doi.org/10.1038/ s41565-021-00872-w

118. J. Min, T. Son, J.S. Hong, P.S. Cheah, A. Wegemann et al., Plasmon-enhanced biosensing for multiplexed profiling of extracellular vesicles. Adv. Biosyst. 4(12), 2000003 (2020). https://doi.org/10.1002/adbi.202000003

119. Q. Su, C. Jiang, D. Gou, Y. Long, Surface plasmon-assisted fluorescence enhancing and quenching: from theory to application. ACS Appl. Bio Mater. 4(6), 4684-4705 (2021). https://doi.org/10.1021/acsabm.1c00320

120. L.K. Chin, T. Son, J.S. Hong, A.Q. Liu, J. Skog et al., Plasmonic sensors for extracellular vesicle analysis: from scientific development to translational research. ACS Nano 14(11), 14528-14548 (2020). https://doi.org/10.1021/acsnano.0c075 81

121. H. Aoki, R.M. Corn, B. Matthews, Microrna detection on microsensor arrays by SPR imaging measurements with enzymatic signal enhancement. Biosens. Bioelectron. 142, 111565 (2019). https://doi.org/10.1016/j.bios.2019.111565

122. C. Zhou, H. Zou, C. Sun, D. Ren, J. Chen et al., Signal amplification strategies for DNA-based surface plasmon resonance biosensors. Biosensors Bioelectron. 117, 678-689 (2018). https://doi.org/10.1016/j.bios.2018.06.062

123. M. Jørgensen, R. Bæk, S. Pedersen, E.K. Søndergaard, S.R. Kristensen et al., Extracellular vesicle (EV) array: microarray capturing of exosomes and other extracellular vesicles for multiplexed phenotyping. J. Extracell. Vesicles 2(1), 20920 (2013). https://doi.org/10.3402/jev.v2i0.20920

124. M.P. Zaborowski, K. Lee, Y.J. Na, A. Sammarco, X. Zhang et al., Methods for systematic identification of membrane proteins for specific capture of cancer-derived extracellular vesicles. Cell Rep. 27(1), 255-268.e6 (2019). https://doi.org/ 10.1016/j.celrep.2019.03.003

125. M.M. Jørgensen, R. Bæk, K. Varming, Potentials and capabilities of the extracellular vesicle (EV) array. J. Extracell. Vesicles 4(1), 26048 (2015). https://doi.org/10.3402/jev.v4. 26048

126. L. Xu, N. Shoaei, F. Jahanpeyma, J. Zhao, M. Azimzadeh et al., Optical, electrochemical and electrical (Nano) biosensors for detection of exosomes: a comprehensive overview. Biosens. Bioelectron. 161, 112222 (2020). https://doi.org/10. 1016/j.bios.2020.112222

127. H. Xu, C. Liao, P. Zuo, Z. Liu, B.C. Ye, Magnetic-based microfluidic device for on-chip isolation and detection of tumor-derived exosomes. Anal. Chem. 90(22), 13451-13458 (2018). https://doi.org/10.1021/acs.analchem.8b03272

128. F. Tian, C. Liu, L. Lin, Q. Chen, J. Sun, Microfluidic analysis of circulating tumor cells and tumor-derived extracellular vesicles. TrAC Trends Anal. Chem. 117, 128-145 (2019). https://doi.org/10.1016/j.trac.2019.05.013

129. J. Park, H.Y. Lin, J.P. Assaker, S. Jeong, C.H. Huang et al., Integrated kidney exosome analysis for the detection of kidney transplant rejection. ACS Nano 11(11), 11041-11046 (2017). https://doi.org/10.1021/acsnano.7b05083

130. C.K. Tang, A. Vaze, M. Shen, J.F. Rusling, High-throughput electrochemical microfluidic immunoarray for multiplexed detection of cancer biomarker proteins. ACS Sens. 1(8), 1036-1043 (2016). https://doi.org/10.1021/acssensors.6b002 56

131. T. Goda, K. Masuno, J. Nishida, N. Kosaka, T. Ochiya et al., A label-free electrical detection of exosomal micrornas using microelectrode array. Chem. Comm. 48(98), 11942-11944 (2012). https://doi.org/10.1039/C2CC36111F

132. C. Jiang, M.T. Alam, S.M. Silva, S. Taufik, S. Fan et al., Unique sensing interface that allows the development of an electrochemical immunosensor for the detection of tumor necrosis factor $\alpha$ in whole blood. ACS Sens. 1(12), 14321438 (2016). https://doi.org/10.1021/acssensors.6b00532

133. Y. Fu, C. Jiang, G.K. Tofaris, J.J. Davis, Facile impedimetric analysis of neuronal exosome markers in Parkinson's disease diagnostics. Anal. Chem. 92(20), 13647-13651 (2020). https://doi.org/10.1021/acs.analchem.0c03092

134. C. Jiang, S.M. Silva, S. Fan, Y. Wu, M.T. Alam et al., Aryldiazonium salt derived mixed organic layers: from surface chemistry to their applications. J. Electroanal. Chem. 785, 265-278 (2017). https://doi.org/10.1016/j.jelechem.2016.11. 043

135. C. Jiang, Protein-Resistant Electrode for Biosensing. PhD Thesis (2016).

136. C. Jiang, M.T. Alam, S.G. Parker, J.J. Gooding, Zwitterionic phenyl phosphorylcholine on indium tin oxide: a lowimpedance protein-resistant platform for biosensing. Electroanalysis 27(4), 884-889 (2015). https://doi.org/10.1002/ elan.201400557

137. S.B. Patil, R.M. Al-Jehani, H. Etayash, V. Turbe, K. Jiang et al., Modified cantilever arrays improve sensitivity and 
reproducibility of nanomechanical sensing in living cells. Commun. Biol. 1(1), 175 (2018). https://doi.org/10.1038/ s42003-018-0179-3

138. J.L. Arlett, E.B. Myers, M.L. Roukes, Comparative advantages of mechanical biosensors. Nat. Nanotechnol. 6(4), 203-215 (2011). https://doi.org/10.1038/nnano.2011.44

139. S. Olcum, N. Cermak, S.C. Wasserman, K.S. Christine, H. Atsumi et al., Weighing nanoparticles in solution at the attogram scale. PNAS 111(4), 1310-1315 (2014). https://doi.org/ 10.1073/pnas.1318602111

140. H. Etayash, A. McGee, K. Kaur, T. Thundat, Nanomechanical sandwich assay for multiple cancer biomarkers in breast cancer cell-derived exosomes. Nanoscale 8(33), 15137-15141 (2016). https://doi.org/10.1039/C6NR03478K

141. G.G. Daaboul, P. Gagni, L. Benussi, P. Bettotti, M. Ciani et al., Digital detection of exosomes by interferometric imaging. Sci. Rep. 6, 37246 (2016). https://doi.org/10.1038/srep3 7246

142. M. Oliveira-Rodríguez, E. Serrano-Pertierra, A.C. García, S. López-Martín, M. Yañez-Mo et al., Point-of-care detection of extracellular vesicles: sensitivity optimization and multipletarget detection. Biosens. Bioelectron. 87, 38-45 (2017). https://doi.org/10.1016/j.bios.2016.08.001

143. G.G. Daaboul, C.A. Lopez, J. Chinnala, B.B. Goldberg, J.H. Connor et al., Digital sensing and sizing of vesicular stomatitis virus pseudotypes in complex media: a model for Ebola and Marburg detection. ACS Nano 8(6), 6047-6055 (2014). https://doi.org/10.1021/nn501312q

144. P. Gebauer, Z. Malá, P. Boček, Recent progress in analytical capillary ITP. Electrophoresis 30(1), 29-35 (2009). https:// doi.org/10.1002/elps.200800425

145. S. Guo, J. Xu, A.P. Estell, C.F. Ivory, D. Du et al., Paperbased ITP technology: an application to specific cancerderived exosome detection and analysis. Biosens. Bioelectron. 164, 112292 (2020). https://doi.org/10.1016/j.bios.2020. 112292

146. V. Shirshahi, G. Liu, Enhancing the analytical performance of paper lateral flow assays: from chemistry to engineering. TrAC Trends Anal. Chem. 136, 116200 (2021). https://doi. org/10.1016/j.trac.2021.116200

147. M. Baharfar, M. Rahbar, M. Tajik, G. Liu, Engineering strategies for enhancing the performance of electrochemical paperbased analytical devices. Biosens. Bioelectron. 167, 112506 (2020). https://doi.org/10.1016/j.bios.2020.112506

148. L. Liu, D. Yang, G. Liu, Signal amplification strategies for paper-based analytical devices. Biosens. Bioelectron. 136, 60-75 (2019). https://doi.org/10.1016/j.bios.2019.04.043

149. Z. Luo, T. Lv, K. Zhu, Y. Li, L. Wang et al., Paper-based ratiometric fluorescence analytical devices towards point-ofcare testing of human serum albumin. Angew. Chem. Int. Ed. 59(8), 3131-3136 (2020). https://doi.org/10.1002/anie.20191 5046

150. C. Jiang, M.T. Alam, S.G. Parker, N. Darwish, J.J. Gooding, Strategies to achieve control over the surface ratio of two different components on modified electrodes using aryldiazonium salts. Langmuir 32(10), 2509-2517 (2016). https://doi.org/10.1021/acs.langmuir.5b04550

151. X. Zhang, Q. Li, X. Jin, C. Jiang, Y. Lu et al., Quantitative determination of target gene with electrical sensor. Sci. Rep. 5(1), 12539 (2015). https://doi.org/10.1038/srep12539

152. S. Zhang, C. Jiang, L. Jia, Tetrabutylammonium phosphateassisted separation of multiplex polymerase chain reaction products in non-gel sieving capillary electrophoresis. Anal. Biochem. 408(2), 284-288 (2011). https://doi.org/10.1016/j. ab.2010.09.022

153. C. Jiang, S. Xu, S. Zhang, L. Jia, Chitosan functionalized magnetic particle-assisted detection of genetically modified soybeans based on polymerase chain reaction and capillary electrophoresis. Anal. Biochem. 420(1), 20-25 (2012). https://doi.org/10.1016/j.ab.2011.09.004

154. C. Song, W. Chen, J. Kuang, Y. Yao, S. Tang et al., Recent advances in the detection of multiple micrornas. TrAC Trends Anal. Chem. 139, 116269 (2021). https://doi.org/10.1016/j. trac. 2021.116269

155. N. Singh, L. Huang, D.B. Wang, N. Shao, X.E. Zhang, Simultaneous detection of a cluster of differentiation markers on leukemia-derived exosomes by multiplex immuno-polymerase chain reaction via capillary electrophoresis analysis. Anal. Chem. 92(15), 10569-10577 (2020). https://doi.org/ 10.1021/acs.analchem.0c01464

156. S.K. Jha, R. Chand, D. Han, Y.C. Jang, G.S. Ra et al., An integrated PCR microfluidic chip incorporating aseptic electrochemical cell lysis and capillary electrophoresis amperometric DNA detection for rapid and quantitative genetic analysis. Lab Chip 12(21), 4455-4464 (2012). https://doi.org/10.1039/ C2LC40727B

157. J. Ko, Y. Wang, J.C.T. Carlson, A. Marquard, J. Gungabeesoon et al., Single extracellular vesicle protein analysis using immuno-droplet digital polymerase chain reaction amplification. Adv. Biosyst. 4(12), 1900307 (2020). https:// doi.org/10.1002/adbi.201900307

158. Q. Tian, C. He, G. Liu, Y. Zhao, L. Hui et al., Nanoparticle counting by microscopic digital detection: selective quantitative analysis of exosomes via surface-anchored nucleic acid amplification. Anal. Chem. 90(11), 6556-6562 (2018). https://doi.org/10.1021/acs.analchem.8b00189

159. J. Ko, Y. Wang, K. Sheng, D.A. Weitz, R. Weissleder, Sequencing-based protein analysis of single extracellular vesicles. ACS Nano 15(3), 5631-5638 (2021). https://doi. org/10.1021/acsnano.1c00782

160. M. Gaňová, H. Zhang, H. Zhu, M. Korabečná, P. Neužil, Multiplexed digital polymerase chain reaction as a powerful diagnostic tool. Biosens. Bioelectron. 181, 113155 (2021). https://doi.org/10.1016/j.bios.2021.113155

161. R. Jara-Acevedo, C. Campos-Silva, M. Valés-Gómez, M. Yáñez-Mó, H. Suárez et al., Exosome beads array for multiplexed phenotyping in cancer. J. Proteomics 198, 87-97 (2019). https://doi.org/10.1016/j.jprot.2018.12.023

162. N. Koliha, Y. Wiencek, U. Heider, C. Jüngst, N. Kladt et al., A novel multiplex bead-based platform highlights the 
diversity of extracellular vesicles. J. Extracell. Vesicles 5(1), 29975 (2016). https://doi.org/10.3402/jev.v5.29975

163. M. Shi, C. Liu, T.J. Cook, K.M. Bullock, Y. Zhao et al., Plasma exosomal $\alpha$-synuclein is likely CNS-derived and increased in Parkinson's disease. Acta Neuropathol. 128(5), 639-650 (2014). https://doi.org/10.1007/s00401-014-1314-y

164. O.P.B. Wiklander, R.B. Bostancioglu, J.A. Welsh, A.M. Zickler, F. Murke et al., Systematic methodological evaluation of a multiplex bead-based flow cytometry assay for detection of extracellular vesicle surface signatures. Front. Immunol. 9(1326), 1326 (2018). https://doi.org/10.3389/fimmu.2018. 01326

165. E. Vacchi, J. Burrello, D. Di Silvestre, A. Burrello, S. Bolis et al., Immune profiling of plasma-derived extracellular vesicles identifies parkinson disease. Neurol. Neuroimmunol. 7(6), e866 (2020). https://doi.org/10.1212/nxi.0000000000 000866

166. G. Marcoux, A.C. Duchez, N. Cloutier, P. Provost, P.A. Nigrovic et al., Revealing the diversity of extracellular vesicles using high-dimensional flow cytometry analyses. Sci. Rep. 6(1), 35928 (2016). https://doi.org/10.1038/srep35928

167. L. Ma, S. Zhu, Y. Tian, W. Zhang, S. Wang et al., Label-free analysis of single viruses with a resolution comparable to that of electron microscopy and the throughput of flow cytometry. Angew. Chem. Int. Ed. 128(35), 10395-10399 (2016). https:// doi.org/10.1002/anie.201603007

168. Y. Tian, L. Ma, M. Gong, G. Su, S. Zhu et al., Protein profiling and sizing of extracellular vesicles from colorectal cancer patients via flow cytometry. ACS Nano 12(1), 671-680 (2018). https://doi.org/10.1021/acsnano.7b07782

169. W.C.W. Chan, D.J. Maxwell, X. Gao, R.E. Bailey, M. Han et al., Luminescent quantum dots for multiplexed biological detection and imaging. Curr. Opin. Biotechnol. 13(1), 40-46 (2002). https://doi.org/10.1016/S0958-1669(02) 00282-3

170. X. Cheng, S.B. Lowe, P.J. Reece, J.J. Gooding, Colloidal silicon quantum dots: from preparation to the modification of self-assembled monolayers (SAMs) for bio-applications. Chem. Soc. Rev. 43(8), 2680-2700 (2014). https://doi.org/ 10.1039/C3CS60353A

171. X. Cheng, E. Hinde, D.M. Owen, S.B. Lowe, P.J. Reece et al., Enhancing quantum dots for bioimaging using advanced surface chemistry and advanced optical microscopy: application to silicon quantum dots (SiQDs). Adv. Mater. 27(40), 6144-6150 (2015). https://doi.org/10.1002/adma.201503223

172. T.A. Taton, G. Lu, C.A. Mirkin, Two-color labeling of oligonucleotide arrays via size-selective scattering of nanoparticle probes. J. Am. Chem. Soc. 123(21), 5164-5165 (2001). https://doi.org/10.1021/ja0102639

173. G.D. Noto, A. Bugatti, A. Zendrini, E.L. Mazzoldi, A. Montanelli et al., Merging colloidal nanoplasmonics and surface plasmon resonance spectroscopy for enhanced profiling of multiple myeloma-derived exosomes. Biosens. Bioelectron. 77, 518-524 (2016). https://doi.org/10.1016/j.bios.2015.09. 061
174. R. Rica, M.M. Stevens, Plasmonic elisa for the ultrasensitive detection of disease biomarkers with the naked eye. Nat. Nanotechnol. 7(12), 821-824 (2012). https://doi.org/10.1038/ nnano.2012.186

175. Y. Zheng, A.H. Soeriyadi, L. Rosa, S.H. Ng, U. Bach et al., Reversible gating of smart plasmonic molecular traps using thermoresponsive polymers for single-molecule detection. Nat. Commun. 6(1), 8797 (2015). https://doi.org/10.1038/ ncomms 9797

176. Y. Zheng, T. Thai, P. Reineck, L. Qiu, Y. Guo et al., DNAdirected self-assembly of core-satellite plasmonic nanostructures: a highly sensitive and reproducible near-IR SERS sensor. Adv. Funct. Mater. 23(12), 1519-1526 (2013). https:// doi.org/10.1002/adfm.201202073

177. K. Liang, F. Liu, J. Fan, D. Sun, C. Liu et al., Nanoplasmonic quantification of tumour-derived extracellular vesicles in plasma microsamples for diagnosis and treatment monitoring. Nat. Biomed. Eng. 1(4), 0021 (2017). https://doi.org/10. 1038/s41551-016-0021

178. Y. Zhao, Z. Xie, H. Gu, C. Zhu, Z. Gu, Bio-inspired variable structural color materials. Chem. Soc. Rev. 41(8), 3297-3317 (2012). https://doi.org/10.1039/C2CS15267C

179. Y.G. Zhou, R.M. Mohamadi, M. Poudineh, L. Kermanshah, S. Ahmed et al., Interrogating circulating microsomes and exosomes using metal nanoparticles. Small 12(6), 727-732 (2016). https://doi.org/10.1002/smll.201502365

180. Y. Wan, Y.G. Zhou, M. Poudineh, T.S. Safaei, R.M. Mohamadi et al., Highly specific electrochemical analysis of cancer cells using multi-nanoparticle labeling. Angew. Chem. Int. Ed. 53(48), 13145-13149 (2014). https://doi.org/10.1002/ anie.201407982

181. H. Wei, S. Ni, C. Cao, G. Yang, G. Liu, Graphene oxide signal reporter based multifunctional immunosensing platform for amperometric profiling of multiple cytokines in serum. ACS Sens. 3(8), 1553-1561 (2018). https://doi.org/ 10.1021/acssensors.8b00365

182. Z. Shen, J. Huang, H. Wei, H. Niu, B. Li et al., Validation of an in vivo electrochemical immunosensing platform for simultaneous detection of multiple cytokines in Parkinson's disease mice model. Bioelectrochemistry 134, 107532 (2020). https://doi.org/10.1016/j.bioelechem.2020.107532

183. W. Guo, H. Ding, C. Gu, Y. Liu, X. Jiang et al., Potentialresolved multicolor electrochemiluminescence for multiplex immunoassay in a single sample. J. Am. Chem. Soc. 140(46), 15904-15915 (2018). https://doi.org/10.1021/jacs. 8b09422

184. W. Lv, H. Ye, Z. Yuan, X. Liu, X. Chen et al., Recent advances in electrochemiluminescence-based simultaneous detection of multiple targets. TrAC Trends Anal. Chem. 123, 115767 (2020). https://doi.org/10.1016/j.trac.2019.115767

185. Y. Bai, Y. Lu, K. Wang, Z. Cheng, Y. Qu et al., Rapid isolation and multiplexed detection of exosome tumor markers via queued beads combined with quantum dots in a microarray. Nano-Micro Lett. 11, 59 (2019). https://doi.org/10.1007/ s40820-019-0285-X 
186. N. Soda, B.H.A. Rehm, P. Sonar, N.T. Nguyen, M.J.A. Shiddiky, Advanced liquid biopsy technologies for circulating biomarker detection. J. Mater. Chem. B 7(43), 6670-6704 (2019). https://doi.org/10.1039/C9TB01490J

187. Q. Guo, Y. Wang, C. Chen, D. Wei, J. Fu et al., Multiplexed luminescence oxygen channeling immunoassay based on dual-functional barcodes with a host-guest structure: a facile and robust suspension array platform. Small 16(17), 1907521 (2020). https://doi.org/10.1002/smll.201907521

188. F. Bian, L. Sun, L. Cai, Y. Wang, Y. Zhao, Bioinspired Mxene-integrated colloidal crystal arrays for multichannel bioinformation coding. PNAS 117(37), 22736-22742 (2020). https://doi.org/10.1073/pnas.2011660117

189. M. Yang, Y. Liu, X. Jiang, Barcoded point-of-care bioassays. Chem. Soc. Rev. 48(3), 850-884 (2019). https://doi.org/10. 1039/C8CS00303C

190. Y. Geng, W.J. Peveler, V.M. Rotello, Array-based "chemical nose" sensing in diagnostics and drug discovery. Angew. Chem. Int. Ed. 58(16), 5190-5200 (2019). https://doi.org/10. 1002/anie.201809607

191. C.C. You, O.R. Miranda, B. Gider, P.S. Ghosh, I.B. Kim et al., Detection and identification of proteins using nanoparticle-fluorescent polymer 'chemical nose' sensors. Nat. Nanotechnol. 2(5), 318-323 (2007). https://doi.org/10.1038/ nnano.2007.99

192. T. Yu, Y. Xianyu, Array-based biosensors for bacteria detection: from the perspective of recognition. Small 17(21), 2006230 (2021). https://doi.org/10.1002/smll.202006230

193. H. Liu, L.H. Xiong, R.T.K. Kwok, X. He, J.W.Y. Lam et al., AIE bioconjugates for biomedical applications. Adv. Opt. Mater. 8(14), 2000162 (2020). https://doi.org/10.1002/adom. 202000162

194. M.T. Yaraki, M. Wu, E. Middha, W. Wu, S.D. Rezaei et al., Gold nanostars-AIE theranostic nanodots with enhanced fluorescence and photosensitization towards effective image-guided photodynamic therapy. Nano-Micro Lett. 13, 58 (2021). https://doi.org/10.1007/s40820-020-00583-2

195. Y. Li, S. Li, J. Wang, G. Liu, Crispr/cas systems towards next-generation biosensing. Trends Biotechnol. 37(7), 730 743 (2019). https://doi.org/10.1016/j.tibtech.2018.12.005

196. Y. Li, L. Liu, G. Liu, CRISPR/Cas multiplexed biosensing: a challenge or an insurmountable obstacle? Trends Biotechnol. 37(8), 792-795 (2019). https://doi.org/10.1016/j.tibte ch.2019.04.012

197. L. Hao, R.T. Zhao, C. Ngambenjawong, H.E. Fleming, S.N. Bhatia, CRISPR-Cas-amplified urine biomarkers for multiplexed and portable cancer diagnostics. bioRxiv (2020). https://doi.org/10.1101/2020.06.17.157180

198. Y. Dai, Y. Wu, G. Liu, J.J. Gooding, CRISPR mediated biosensing toward understanding cellular biology and point-of-care diagnosis. Angew. Chem. Int. Ed. 59(47), 20754-20766 (2020). https://doi.org/10.1002/anie.20200 5398

199. J.S. Gootenberg, O.O. Abudayyeh, M.J. Kellner, J. Joung, J.J. Collins et al., Multiplexed and portable nucleic acid detection platform with Cas13, Cas12a, and Csm6. Science 360(6387), 439-444 (2018). https://doi.org/10.1126/science.aaq0179

200. H. Yue, B. Shu, T. Tian, E. Xiong, M. Huang et al., Droplet Cas12a assay enables DNA quantification from unamplified samples at the single-molecule level. Nano Lett. 21(11), 4643-4653 (2021). https://doi.org/10.1021/acs.nanolett. $1 \mathrm{c} 00715$

201. C.M. Ackerman, C. Myhrvold, S.G. Thakku, C.A. Freije, H.C. Metsky et al., Massively multiplexed nucleic acid detection with Cas13. Nature 582(7811), 277-282 (2020). https:// doi.org/10.1038/s41586-020-2279-8

202. N.E. Weckman, N. Ermann, R. Gutierrez, K. Chen, J. Graham et al., Multiplexed DNA identification using site specific dCas9 barcodes and nanopore sensing. ACS Sens. 4(8), 2065-2072 (2019). https://doi.org/10.1021/acssensors.9b006 86

203. R. Bruch, G.A. Urban, C. Dincer, Crispr/Cas powered multiplexed biosensing. Trends Biotechnol. 37(8), 791-792 (2019). https://doi.org/10.1016/j.tibtech.2019.04.005

204. R. Bruch, J. Baaske, C. Chatelle, W. Weber, C. Dincer et al. Electrochemical biosensor for Crispr/Cas13a powered miRNA diagnostics. 2019 IEEE SENSORS, Montreal, QC, Canada, 27-30 Oct. (2019). https://ieeexplore.ieee.org/docum ent/8956561

205. F. Mei, S.P.J. Fancy, Y.A.A. Shen, J. Niu, C. Zhao et al., Micropillar arrays as a high-throughput screening platform for therapeutics in multiple sclerosis. Nat. Med. 20(8), 954960 (2014). https://doi.org/10.1038/nm.3618

206. P. Zhang, X. Wu, G. Gardashova, Y. Yang, Y. Zhang et al., Molecular and functional extracellular vesicle analysis using nanopatterned microchips monitors tumor progression and metastasis. Sci. Transl. Med. 12(547), 2878 (2020). https:// doi.org/10.1126/scitranslmed.aaz2878

207. P. Zhang, X. Zhou, M. He, Y. Shang, A.L. Tetlow et al., Ultrasensitive detection of circulating exosomes with a 3D-nanopatterned microfluidic chip. Nat. Biomed. Eng. 3(6), 438-451 (2019). https://doi.org/10.1038/s41551-019-0356-9

208. L. Huang, S. Tian, W. Zhao, K. Liu, X. Ma et al., Multiplexed detection of biomarkers in lateral-flow immunoassays. Analyst 145(8), 2828-2840 (2020). https://doi.org/10.1039/ C9AN02485A

209. W. Zhao, S. Tian, L. Huang, K. Liu, L. Dong et al., A smartphone-based biomedical sensory system. Analyst 145(8), 2873-2891 (2020). https://doi.org/10.1039/C9AN02294E

210. C. Dincer, R. Bruch, A. Kling, P.S. Dittrich, G.A. Urban, Multiplexed point-of-care testing-Xpoct. Trends Biotechnol. 35(8), 728-742 (2017). https://doi.org/10.1016/j.tibte ch. 2017.03 .013

211. J. Kaur, C. Jiang, G. Liu, Different strategies for detection of HBA1C emphasizing on biosensors and point-of-care analyzers. Biosens. Bioelectron. 123(1), 85-100 (2019). https://doi. org/10.1016/j.bios.2018.06.018

212. Z. Dong, C. Tang, Z. Zhang, W. Zhou, R. Zhao et al., Simultaneous detection of exosomal membrane protein and RNA by highly sensitive aptamer assisted multiplex-PCR. ACS 
Appl. Bio Mater. 3(5), 2560-2567 (2020). https://doi.org/10. 1021/acsabm.9b00825

213. C.Z.J. Lim, A. Natalia, N.R. Sundah, H. Shao, Biomarker organization in circulating extracellular vesicles: new applications in detecting neurodegenerative diseases. Adv. Biosyst. 4(12), 1900309 (2020). https://doi.org/10.1002/adbi.20190 0309

214. K.E. Richards, D.B. Go, R. Hill (2017) Surface acoustic wave lysis and ion-exchange membrane quantification of exosomal microRNA. MicroRNA Detection and Target Identification. Methods in Molecular Biology chapter 5, 59-70. https://doi. org/10.1007/978-1-4939-6866-4_5

215. D. Taller, K. Richards, Z. Slouka, S. Senapati, R. Hill et al., On-chip surface acoustic wave lysis and ion-exchange nanomembrane detection of exosomal RNA for pancreatic cancer study and diagnosis. Lab Chip 15(7), 1656-1666 (2015). https://doi.org/10.1039/C5LC00036J

216. F. Zhu, Y. Ji, J. Deng, L. Li, X. Bai et al., Microfluidics-based technologies for the analysis of extracellular vesicles at the single-cell level and single-vesicle level. Chin. Chem. Lett. (2021). https://doi.org/10.1016/j.cclet.2021.09.058

217. Y. Orooji, H. Sohrabi, N. Hemmat, F. Oroojalian, B. Baradaran et al., An overview on SARS-Cov-2 (COVID-19) and other human coronaviruses and their detection capability via amplification assay, chemical sensing, biosensing, immunosensing, and clinical assays. Nano-Micro Lett. 13, 18 (2020). https://doi.org/10.1007/s40820-020-00533-y
218. C. Cao, Y. Zhang, C. Jiang, M. Qi, G. Liu, Advances on aryldiazonium salt chemistry based interfacial fabrication for sensing applications. ACS Appl. Mater. Interfaces 9(6), 5031-5049 (2017). https://doi.org/10.1021/acsami.6b16108

219. A. Krug, D. Enderle, C. Karlovich, T. Priewasser, S. Bentink et al., Improved EGFR mutation detection using combined exosomal RNA and circulating tumor DNA in NSCLC patient plasma. Ann. Oncol. 29(3), 700-706 (2018). https://doi.org/ 10.1093/annonc/mdx765

220. H. Shin, S. Oh, S. Hong, M. Kang, D. Kang et al., Early-stage lung cancer diagnosis by deep learning-based spectroscopic analysis of circulating exosomes. ACS Nano 14(5), 54355444 (2020). https://doi.org/10.1021/acsnano.9b09119

221. J. Riordon, D. Sovilj, S. Sanner, D. Sinton, E.W. Young, Deep learning with microfluidics for biotechnology. Trends Biotechnol. 37(3), 310-324 (2019). https://doi.org/10.1016/j. tibtech.2018.08.005

222. L. Min, B. Wang, H. Bao, X. Li, L. Zhao et al., Advanced nanotechnologies for extracellular vesicle-based liquid biopsy. Adv. Sci. 8(20), 2102789 (2021). https://doi.org/10. 1002/advs.202102789

223. K. Fraser, A. Jo, J. Giedt, C. Vinegoni, K.S. Yang et al., Characterization of single microvesicles in plasma from glioblastoma patients. Neuro Oncol. 21(5), 606-615 (2019). https:// doi.org/10.1093/neuonc/noy187 